University of Nebraska - Lincoln

DigitalCommons@University of Nebraska - Lincoln

\title{
Environmental contaminants and biomarker responses in fish from the Rio Grande and its U.S. tributaries: Spatial and temporal trends
}

\author{
Christopher J. Schmitt \\ U.S. Geological Survey, cjschmitt@usgs.gov \\ Jo Ellen Hinck \\ Columbia Environmental Research Center, jhinck@usgs.gov \\ Vicki S. Blazer \\ USGS Leetown Science Center \\ Nancy D. Denslow \\ University of Florida, denslown@vetmed.ufl.edu \\ Gail M. Dethloff \\ AScl, gmdethloff@ucdavis.edu \\ See next page for additional authors
}

Follow this and additional works at: https://digitalcommons.unl.edu/usgsstaffpub

Schmitt, Christopher J.; Hinck, Jo Ellen; Blazer, Vicki S.; Denslow, Nancy D.; Dethloff, Gail M.; Bartish, Timothy M.; Coyle, James J.; and Tillitt, Donald E., "Environmental contaminants and biomarker responses in fish from the Rio Grande and its U.S. tributaries: Spatial and temporal trends" (2005). USGS Staff -Published Research. 568.

https://digitalcommons.unl.edu/usgsstaffpub/568

This Article is brought to you for free and open access by the US Geological Survey at DigitalCommons@University of Nebraska - Lincoln. It has been accepted for inclusion in USGS Staff -- Published Research by an authorized administrator of DigitalCommons@University of Nebraska - Lincoln. 


\section{Authors}

Christopher J. Schmitt, Jo Ellen Hinck, Vicki S. Blazer, Nancy D. Denslow, Gail M. Dethloff, Timothy M.

Bartish, James J. Coyle, and Donald E. Tillitt 


\title{
Environmental contaminants and biomarker responses in fish from the Rio Grande and its U.S. tributaries: Spatial and temporal trends
}

\author{
Christopher J. Schmitt ${ }^{\mathrm{a}, *}$, Jo Ellen Hinck ${ }^{\mathrm{a}}$, Vicki S. Blazer ${ }^{\mathrm{b}}$, Nancy D. Denslow ${ }^{\mathrm{c}}$, \\ Gail M. Dethloff $^{\mathrm{d}, 1}$, Timothy M. Bartish ${ }^{\mathrm{e}}$, James J. Coyle ${ }^{\mathrm{e}}$, Donald E. Tillitt ${ }^{\mathrm{a}}$ \\ ${ }^{a}$ U.S. Geological Survey (USGS), Columbia Environmental Research Center (CERC), 4200 New Haven Rd., Columbia, MO 65201, USA \\ ${ }^{\mathrm{b}}$ USGS Leetown Science Center, 11649 Leetown Rd., Kearneysville, WV 24530-9407, USA \\ ${ }^{\mathrm{c}}$ Protein Chemistry and Molecular Biomarkers Laboratory, PO Box 100156 Health Center, University of Florida, Gainesville, FL 32610, USA \\ ${ }^{\mathrm{d}} A S C I$, clo USGS-CERC, USA \\ ${ }^{\mathrm{e}}$ USGS, Office of the Chief Science-Biologist, 12201 Sunrise Valley Dr. MS-300, Reston, VA 20192, USA
}

Received 21 October 2004; accepted 12 January 2005

Available online 17 March 2005

\begin{abstract}
We collected, examined, and analyzed 368 fish of seven species from 10 sites on rivers of the Rio Grande Basin (RGB) during late 1997 and early 1998 to document temporal and geographic trends in the concentrations of accumulative contaminants and to assess contaminant effects on the fish. Sites were located on the mainstem of the Rio Grande and on the Arroyo Colorado and Pecos River in Texas (TX), New Mexico (NM), and Colorado. Common carp (Cyprinus carpio) and largemouth bass (Micropterus salmoides) were the targeted species. Fish were examined in the field for internal and external visible gross lesions, selected organs were weighed to compute ponderal and organosomatic indices, and samples of tissues and fluids were obtained and preserved for analysis of fish health and reproductive biomarkers. Whole fish from each station were composited by species and gender and analyzed for organochlorine chemical residues and elemental contaminants using instrumental methods, and for 2,3,7,8-tetrachloro dibenzo- $p$-dioxin-like activity (TCDD-EQ) using the H4IIE rat hepatoma cell bioassay. Overall, fish from lower RGB stations contained greater concentrations of organochlorine pesticide residues and appeared to be less healthy than those from sites in the central and upper parts of the basin, as indicated by a general gradient of residue concentrations and biomarker responses. A minimal number of altered biomarkers and few or no elevated contaminant concentrations were noted in fish from the upper RGB. The exception was elevated concentrations [up to $0.46 \mu \mathrm{g} / \mathrm{g}$ wet-weight $(\mathrm{ww})$ ] of total mercury ( $\mathrm{Hg}$ ) in predatory species from the Rio Grande at Elephant Butte Reservoir, NM, a condition documented in previous studies. Arsenic (As) and selenium (Se) concentrations were greatest in fish from sites in the central RGB; Se concentrations in fish from the Pecos River at Red Bluff Lake, TX and from the Rio Grande at Langtry, TX and Amistad International Reservoir, TX exceeded published fish and wildlife toxicity thresholds. In the lower RGB, residues of
\end{abstract}

\footnotetext{
* Corresponding author.

E-mail address: cjschmitt@usgs.gov (C.J. Schmitt).

${ }^{1}$ Present address: ENSR International, 4303 W. LaPorte Ave., Fort Collins, CO 80521-2154, USA.
} 
$p, p^{\prime}$-DDT metabolites $(\leq 1.69 \mu \mathrm{g} / \mathrm{g} w \mathrm{ww})$, chlordane-related compounds $(\leq 0.21 \mu \mathrm{g} / \mathrm{g} w \mathrm{ww})$, dieldrin $(\leq 0.0 .05 \mu \mathrm{g} / \mathrm{g} \mathrm{ww})$, and toxaphene $(\leq 2.4 \mu \mathrm{g} / \mathrm{g} \mathrm{ww})$ were detected in fish from most sites; maximum concentrations were in channel catfish (Ictalurus punctatus) from the Arroyo Colorado at Harlingen, TX. Concentrations of one or more residues exceeded toxicity thresholds for fish and wildlife in fish from this site and from the Rio Grande at Mission, TX and Brownsville, TX; however, concentrations were lower than those reported by previous studies. In addition, the proportional concentrations of $p, p^{\prime}$-DDT at all sites were low, indicating weathered DDT rather than the influx of new material. Concentrations of total PCBs $(<0.05 \mu \mathrm{g} / \mathrm{g} \mathrm{ww})$ and TCDD-EQ ( $\leq 6 \mathrm{pg} / \mathrm{g} \mathrm{ww}$ ) were comparatively low in all samples. Hepatic ethoxyresorufin $O$-deethylase (EROD) activity in some fish was elevated relative to reference rates at most sites, but was generally lower than previously reported activity in fish from heavily contaminated locations. The comparatively low PCB and TCDD-EQ concentrations together with elevated EROD activity may reflect exposure to polycyclic aromatic hydrocarbons. Reproductive biomarkers were consistent with chronic contaminant exposure at lower RGB sites; comparatively large percentages of intersex male largemouth bass, relatively low gonadosomatic indices, and elevated plasma vitellogenin concentrations in male fish were noted at three of the four stations. Large percentages of atretic eggs were also observed in the ovaries of female common carp from the Rio Grande at Brownsville, TX. Although many of the conditions noted may have other causes in addition to contaminant exposure, the biomarker results for the lower RGB sites are consistent with subtle responses of fish to contaminants, an interpretation supported by the chemical data of this and other investigations.

(C) 2005 Published by Elsevier B.V.

Keywords: Arsenic; Selenium; Mercury; Pesticides; Organochlorine chemicals; Ethoxyresorufin $O$-deethylase (EROD) activity; Health assessment index, (HAI); Biomarkers; Ovotestis; Vitellogenin

\section{Introduction}

The Rio Grande is the second longest U.S. river; it is approximately $3059 \mathrm{~km}$ long and drains an area of some $924,300 \mathrm{~km}^{2}$ (Texas Natural Resources Conservation Commission (TNRCC), 1997; Fig. 1). The Rio Grande also represents the international boundary between the United States and Mexico from El Paso, Texas (TX) to the Gulf of Mexico, a distance of about $2053 \mathrm{~km}$ (Fig. 1). About 69\% $\left(231,317 \mathrm{~km}^{2}\right)$ of the Rio Grande Basin (RGB) lies within the United States, with the remainder in Mexico. Although much of the RGB is desert, the area supports a rapidly expanding human population conservatively estimated at 10 million in 1992 (TNRCC, 1997) as well as a unique river-dependent biota. The Rio Grande and its tributaries therefore represent a vital source of water to both the human population and the ecological resources of the region. Heavy demands are placed on the Rio Grande and its tributaries for irrigation and for the water supply and waste disposal needs of population centers in the United States and Mexico. Water quality is affected by natural, agricultural, industrial, and urban erosional processes, which contribute to high sediment loads, and dams and diversions have dramatically altered flow regimes (e.g., Ong et al., 1991; TNRCC, 1994a, 1997;
Davis et al., 1995; Levings et al., 1998). Chemically intensive irrigated agriculture is practiced in much of the RGB, parts of which are also highly mineralized and underlain by petroleum-rich geologic formations. Consequently, pesticides, oil, and potentially toxic trace elements such as arsenic (As), selenium (Se), and heavy metals are available for mobilization and transport. The rates of the processes controlling the release and distribution of these constituents have been profoundly altered by human activities such as irrigation, mining, oil and gas extraction, and complex systems of dams and diversions, which have profoundly affected the flux of water and sediments and their associated contaminants. Elevated concentrations of metals, metalloids, and organochlorine pesticides have been documented in sediments and river-dependent organisms throughout the RGB (Gamble et al., 1988; Ong et al., 1991; U.S. Environmental Protection Agency (USEPA), 1992; TNRCC, 1994a,b, 1997; Davis et al., 1995; Carter and Anderholm, 1997; Mora, 1997; Mora et al., 1997; Van Metre et al., 1997; Mora and Wainwright, 1998; Levings et al., 1998; Schmitt et al., 1999b; Moring, 1999).

We sampled the largest U.S. rivers in the RGB during late 1997 and early 1998 as part of the U.S. Geological Survey (USGS) Biomonitoring of Environmental Status and Trends program, which monitors 


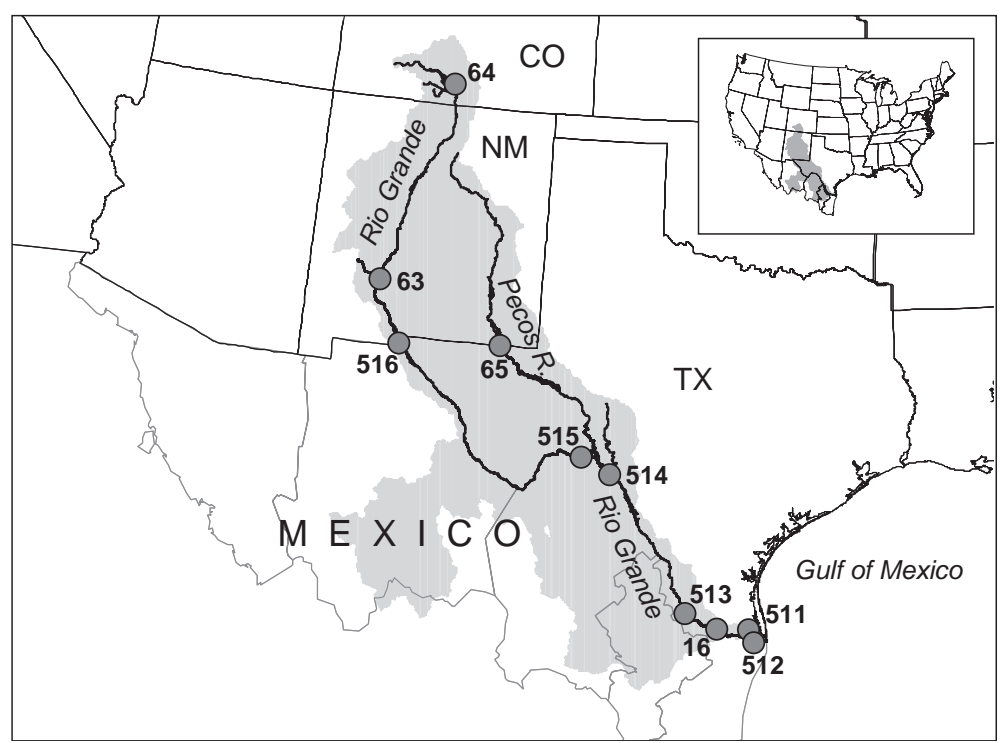

Fig. 1. Map of the Rio Grande basin illustrating waterways and impoundments, state and international boundaries, and locations sampled.

and evaluates environmental contaminants and their effects on fish throughout the United States (Schmitt, 2002a). Our primary objective was to document and assess spatial and temporal trends in the concentrations of environmental contaminants and their effects in RGB fish. Secondary objectives were to compare results from the RGB to other major U.S. rivers, and to further define benchmarks for the quantification of long-term trends and interpretation of biomarker results. In this paper we summarize the most pertinent findings of the RGB study, which are reported in greater detail by Schmitt et al. (2004). All raw data from this and related investigations are available at $<$ http://www.cerc.usgs.gov/data/data.htm>.

\section{Materials and methods}

The chemical and biological methods used to assess exposure of fish to contaminants and the effects of exposure were selected to span multiple levels of biological organization. The methods included exposure indicators [concentrations of elemental contaminants, organochlorine chemical residues, and 2,3,7,8-tetrachloro dibenzo- $p$-dioxin equivalents (TCDD-EQ); and hepatic ethoxyresorufin $O$-deethylase (EROD) activity], fish health indicators [ponderal and somatic indices, external lesions, health assessment index (HAI), and general histopathology], and reproductive biomarkers [gonadosomatic index (GSI), gonadal histopathology, and plasma vitellogenin (vtg) concentrations]. Detailed descriptions of all procedures and quality assurance (QA) results are presented elsewhere (Schmitt et al., 1999a; Schmitt and Dethloff, 2000; Schmitt, 2002a,b, 2004).

\subsection{Sampling and field procedures}

Fish were collected at 10 sites located on the mainstem of the Rio Grande in Colorado (CO), New Mexico (NM), and Texas $(n=8)$; the Arroyo Colorado, a distributary of the Rio Grande, in South Texas $(n=1)$; and the Pecos River, the largest U.S. tributary of the Rio Grande, near the Texas-New Mexico boundary ( $n=1$; Fig. 1 ; Table 1$)$. To ensure spatial and temporal continuity with historical data and facilitate trend analysis, four sites were National Contaminant Biomonitoring Program (NCBP) stations where contaminants in fish had been monitored historically (Schmitt et al., 1999b). Most fish were collected between late September and early December 1997, but Station 65 was not sampled until January 1998 (Table 1). Sampling was completed during one visit spanning 2-3 d at most sites, but two visits were 
Table 1

Location and fish collection dates of sampling stations in the Rio Grande (RG) basin (listed upstream to downstream)

\begin{tabular}{|c|c|c|c|c|}
\hline Sub-basin and river & $\begin{array}{l}\text { Station } \\
\text { number }\end{array}$ & Location & Collection date(s) & Latitude, longitude \\
\hline \multicolumn{5}{|l|}{ Upper $R G B$} \\
\hline RG & $64^{\mathrm{a}}$ & Alamosa, CO & $09 / 23 / 97-10 / 21 / 97$ & $37^{\circ} 25^{\prime} 06.42^{\prime \prime} \mathrm{N}, 105^{\circ} 46^{\prime} 48.48^{\prime \prime} \mathrm{W}$ \\
\hline RG & $63^{\mathrm{a}}$ & Elephant Butte Reservoir, NM & $10 / 22 / 97-10 / 24 / 97$ & $33^{\circ} 12^{\prime} 48.55^{\prime \prime} \mathrm{N}, 107^{\circ} 13^{\prime} 27.26^{\prime \prime} \mathrm{W}$ \\
\hline RG & 516 & El Paso, TX & $10 / 30 / 97-12 / 03 / 97$ & $31^{\circ} 47^{\prime} 55.00^{\prime \prime} \mathrm{N}, 106^{\circ} 32^{\prime} 25.08^{\prime \prime} \mathrm{W}$ \\
\hline \multicolumn{5}{|l|}{ Middle RGB/Pecos } \\
\hline Pecos & $65^{\mathrm{a}}$ & Red Bluff Lake, TX & $01 / 21 / 98-01 / 22 / 98$ & $32^{\circ} 00^{\prime} 00.00^{\prime \prime} \mathrm{N}, 103^{\circ} 58^{\prime} 56.28^{\prime \prime} \mathrm{W}$ \\
\hline $\mathrm{RG}$ & 515 & Foster Ranch, Langtry, TX & $11 / 06 / 97$ & $29^{\circ} 46^{\prime} 40.91^{\prime \prime} \mathrm{N}, 101^{\circ} 45^{\prime} 13.22^{\prime \prime} \mathrm{W}$ \\
\hline RG & 514 & Below Amistad Reservoir, TX & $11 / 04 / 97-11 / 05 / 97$ & $29^{\circ} 26^{\prime} 49.06^{\prime \prime} \mathrm{N}, 101^{\circ} 03^{\prime} 22.44^{\prime \prime} \mathrm{W}$ \\
\hline \multicolumn{5}{|l|}{ Lower $R G B$} \\
\hline RG & 513 & Below Falcon Dam, TX & $11 / 18 / 97-11 / 19 / 97$ & $20^{\circ} 08^{\prime} 06.66^{\prime \prime} \mathrm{N}, 99^{\circ} 08^{\prime} 06.42^{\prime \prime} \mathrm{W}$ \\
\hline RG & $16^{\mathrm{a}}$ & Mission, TX & $12 / 02 / 97-12 / 03 / 97$ & $26^{\circ} 09^{\prime} 28.74^{\prime \prime} \mathrm{N}, 98^{\circ} 20^{\prime} 02.82^{\prime \prime} \mathrm{W}$ \\
\hline Arroyo Colorado & 511 & Harlingen, TX & 09/30/97-10/02/97 & $26^{\circ} 11^{\prime} 44.28^{\prime \prime} \mathrm{N}, 97^{\circ} 36^{\prime} 20.52^{\prime \prime} \mathrm{W}$ \\
\hline RG & 512 & Brownsville, TX & $10 / 28 / 97-11 / 25 / 97$ & $25^{\circ} 52^{\prime} 12.96^{\prime \prime} \mathrm{N}, 97^{\circ} 27^{\prime} 06.30^{\prime \prime} \mathrm{W}$ \\
\hline
\end{tabular}

${ }^{a}$ National Contaminant Biomonitoring Program (NCBP) site (Schmitt et al., 1999b).

required to complete the collections at Stations 64, 512, and 516 (Table 1).

Common carp (Cyprinus carpio, henceforth carp) and largemouth bass (Micropterus salmoides) were the preferred species, with a collection target of 10 (each) males and females of each taxon (total of 40 fish) per site. Adult fish of a size representative of those believed to be present at the sites based on extant information were sought, and extremely large or small fish were avoided.

Fish were collected by electrofishing, with the following exceptions: smallmouth bass (Micropterus dolomieui) were netted at Station 63, as were all fish from Stations 511 and 515 except blue catfish (Ictalurus furcatus), which were captured by hand. Fish were held in aerated live wells and transported to shore for processing, usually within a few hours of collection. Some fish were held alive overnight in aerated tanks containing ambient water or in situ in net pens following night collections. Fish processing began with the collection of a blood sample from the posterior caudal artery and vein with a heparinized needle and syringe. The blood sample was chilled on [wet] ice, and the fish was weighed, measured, and subdued with a blow to the head. Observations of external features were recorded, and grossly visible anomalies were removed by dissection and preserved in $10 \%$ neutral buffered formalin (NBF) for histopathological analysis. The abdominal cavity of the fish was dissected open and the liver (in species other than carp), spleen, and gonads were removed and weighed. The liver, gall bladder, posterior and anterior kidneys, gonads, mesenteric fat, and spleen were examined, and the gender of the fish was determined by gonadal observation. Pieces of liver were collected and immediately flash-frozen in a dry ice-ethanol slush for EROD analysis. Samples of gonad (posterior tips), kidney, spleen, and additional pieces of liver were collected and preserved in $10 \% \mathrm{NBF}$ for histopathological examination, which included gender confirmation (gonad) and macrophage aggregate quantification (spleen). Scales (spines from ictalurids) were collected for age determination. All remaining tissues (those not frozen or fixed) were wrapped in aluminum foil, chilled, and later frozen for analysis of organochlorine chemical residues, elemental contaminants, and TCDD-EQ, the latter using the H4IIE bioassay (Tillitt et al., 1991). Between specimens all contact instruments and work surfaces were thoroughly cleaned to prevent cross-contamination. Following fish processing, blood samples were centrifuged and the plasma was aspirated and frozen in dry ice for vtg analysis. Cryogenically frozen liver and plasma samples were shipped frozen (in nitrogen dry shippers) and stored at $-80{ }^{\circ} \mathrm{C}$. Fish carcasses were shipped frozen in dry ice to the analytical laboratory and stored at $-20{ }^{\circ} \mathrm{C}$ until prepared for analysis. 


\subsection{Laboratory analyses}

Composite carcass samples were prepared for chemical and H4IIE analyses by band-sawing and grinding with a commercial meat grinder. The ground fish comprising each sample was subsampled and refrozen $\left(-20{ }^{\circ} \mathrm{C}\right)$. One subsample $(100 \mathrm{~g})$ was freezedried; following determination of moisture content (as moisture loss during lyophilization), a portion was acid-digested and analyzed for 19 elemental contaminants by atomic absorption spectroscopy and inductively coupled plasma emission spectroscopy. Quality assurance measures for the elemental analyses included the analysis of reagent blanks, duplicate samples, certified reference materials, and fortified samples. Dry-weight (dw) limits-of-detection (LODs) were determined individually for each element in each sample; for the elements reported here the nominal LODs (all dw) were $0.1 \mu \mathrm{g} / \mathrm{g}$ for cadmium (Cd) and total mercury ( $\mathrm{Hg}$ ), $0.2 \mu \mathrm{g} / \mathrm{g}$ for lead (Pb), $0.5 \mu \mathrm{g} / \mathrm{g}$ for As and Se, and $1.5 \mu \mathrm{g} / \mathrm{g}$ for zinc (Zn). For statistical analysis and reporting, all elemental concentrations and LODs were converted to wet-weight (ww) values using the moisture content of each sample. A second subsample (10 g) was solvent-extracted and analyzed gravimetrically for lipid content and by gas chromatography with electron capture detection for 21 organochlorine pesticide residues and total polychlorinated biphenyls (PCBs). Quality assurance measures for the organochlorine analyses included the analysis of duplicate and fortified samples and the confirmation of residue identities in selected samples by gas chromatography-mass spectrometry. Recovery efficiency ranged from $60.2 \%$ for hexachlorobenzene (HCB) to $94.4 \%$ for mirex, but was $85-92 \%$ for most analytes. Nominal LODs were $0.01 \mu \mathrm{g} / \mathrm{g}$ wet-weight (ww) for individual compounds and $0.05 \mu \mathrm{g} / \mathrm{g}$ for toxaphene and total PCBs. Residue concentrations were not adjusted for recovery efficiency. A third subsample ( $10 \mathrm{~g})$ was solvent-extracted and subjected to reactive cleanup for use in the H4IIE bioassay (Tillitt et al., 1991) as modified for 96-well microtiter plates (Tysklind et al., 1994). Concentrations of TCDD-EQ (pg/g ww) were determined by slope ratio assay (Finney, 1980) as described by Ankley et al. (1991). Quality assurance measures for the H4IIE bioassay included the analysis of duplicate samples and reference materials. Limits-of-quantitation
(LOQs) and LODs for TCDD-EQ were computed separately for each group of samples; LODs were 0-1 $\mathrm{pg} / \mathrm{g}$ and all LOQs were $1 \mathrm{pg} / \mathrm{g}$.

Hepatic EROD activity was determined in 96-well microtiter plates using kinetic assays performed on triplicate $5-\mu \mathrm{L}$ portions of microsomal fractions prepared daily from each fish liver sample (Whyte et al., 2000). Protein content was determined using the fluorescamine protein assay (Lorenzen and Kennedy, 1993) in the same 96-well plate as the EROD analyses and used to normalize EROD activity in each well. Activity was reported as the mean of the triplicate determinations. LODs were $0-0.15 \mathrm{pmol} / \mathrm{min} / \mathrm{mg}$; LOQs were $0-0.35 \mathrm{pmol} / \mathrm{min} / \mathrm{mg}$. In addition to the triplicate determinations, QA measures for the EROD assay included the analysis of reference materials and duplicate samples.

Fixed tissues (liver, kidney, spleen, gill, gonad, and grossly visible lesions) were prepared for histopathological analysis as described by Blazer et al. (2002). Paraffin-embedded tissue sections $(6-\mu \mathrm{m})$ mounted on glass slides were stained with hematoxylin and eosin (H and $\mathrm{E})$ for microscopic examination. Macrophage aggregates (MA) and MA pigments in spleen sections were stained using Perl's method (Luna, 1992). All MA measurements were made with a computer-based image analysis system, and included the number of aggregates in 2 $\mathrm{mm}^{2}$ of tissue (MA-\#) and the mean area occupied by those aggregates (MA-A). The percentage of tissue occupied by MA (MA-\%) was computed from these measurements. Transverse ovary sections were assigned to developmental stages $0-5$ based on the predominant size and appearance of oocytes (Rodriguez et al., 1995; Nagahama, 1983; Treasurer and Holliday, 1981; McDonald et al., 2000; Blazer, 2002), and oocyte atresia was quantified. Transverse testes sections were similarly classified into developmental stages 0-4 (Nagahama, 1983; Blazer, 2002). Gonadal tissue was also examined microscopically for any abnormalities such as intersex, parasites, neoplasia, and pigmented cell accumulations (i.e., ceroid/lipofuscin deposits). Pigment deposits were categorized as absent, present, or abundant. Male fish were identified as intersex when individual or small foci of undeveloped oocytes were observed within testicular tissue (i.e., when an ovotestis condition was detected). 
Concentrations of vtg in largemouth and smallmouth bass (henceforth bass) and carp plasma were determined by ELISA (Denslow et al., 1999). The LOD was $0.002 \mathrm{mg} / \mathrm{mL}$ for bass and $0.005 \mathrm{mg} / \mathrm{mL}$ for carp. All assays were performed in triplicate and reported as the mean of the three measurements; the coefficient of variation was $<10 \%$ for all samples analyzed. Inter-assay variability was $<10 \%$ as determined by routinely analyzing controls on several plates.

\subsection{Data set composition and statistical analyses}

A total of 368 fish representing eight species were collected and examined. Carp $(n=207)$ and bass $(n=75)$ together represented $77 \%$ of the total. Carp were obtained at all 10 stations and bass at five; the other species [blue catfish, channel catfish (Ictalurus punctatus), striped bass (Morone saxatilis), white bass (M. chrysops), and northern pike (Esox lucius)] were obtained at only one or two stations. Only three smallmouth bass were collected-two females at Station 63 and one male at Station 514. All other bass were largemouth. Of the species other than carp and bass, only channel catfish $(n=42)$ accounted for more than $10 \%$ of the total. Composite samples $(n=47)$ from 10 stations were analyzed for organochlorine chemical residues, elemental contaminants, and TCDD-EQ. Of these, 22 samples (47\%) representing all ten stations were carp and 12 samples $(25.5 \%)$ from five stations were bass. The remaining 13 samples $(26 \%)$ comprised channel catfish $(n=5$, two stations), northern pike ( $n=4$, one station), striped bass $(n=1$, one station), blue catfish $(n=1$, one station), and white bass ( $n=2$, one station).

The occurrence of gross external pathological disorders was determined by assigning numerical values to field observations. Lesions were rated as present (1) or absent (0). For consistency with previous studies (e.g. Fournie et al., 1996, 2001; Blazer et al., 2002), only the following observations were included: grossly visible disorders of the eye (exopthalmia, hemorrhage, opacity, emboli, missing), opercles (shortening, deformities, parasites), body surface (ulcers, parasites, and raised or discolored areas), fins (hemorrhage, fraying, eroded), and skeleton (curvature). A necropsy-based HAI score was also calculated for each fish by assigning numerical values to gross internal and external lesions (Adams et al., 1993; Blazer et al., 2002), then summing the values for all organs observed. An HAI score was computed for a fish only if observations were present for all components.

Body and organ weights were used to compute condition factor (CF) and organosomatic indices (Dethloff and Schmitt, 2000; Blazer et al., 2002) according to the following formulae: $\mathrm{CF}=$ body weight/length ${ }^{3}$; hepatosomatic index $(\mathrm{HSI})=$ liver weight/(total body weight - gonad weight $) \times 100$; splenosomatic index $(\mathrm{SSI})=$ spleen weight/(total body weight - gonad weight) $\times 100 ; \mathrm{GSI}=$ gonad weight/ total body weight $\times 100$. The weight of the gonads was subtracted from the body weight in the computation of HSI and SSI to minimize the effect of the reproductive cycle on these indices (Dethloff and Schmitt, 2000).

Some fish were grouped at the genus level for statistical analysis. Most biomarker results were analyzed using analysis-of-variance (ANOVA) and analysis-of-covariance (ANCOVA) to test for differences among sites and to examine for effects due to age, gender, and gonadal stage. Least-squares means, which are adjusted for all effects in the model, were tested. Transformations were applied to approximate the normality and homogeneity-of-variance required for the application of these parametric statistical methods. Contaminant concentrations (including TCDD-EQ) in composite samples and EROD activities and vtg concentrations in individual fish (females only for vtg) were $\log _{10}$-transformed; and HAI scores were rank-transformed. The length, weight, and age data were not transformed. External lesion frequencies were not analyzed statistically but were accounted for in the HAI scores. All computations and statistical analyses were performed with Version 8 of the Statistical Analysis System (SAS Institute, 1999).

Fish from which only regenerated scales were collected (22 carp, one largemouth bass) were excluded from all analyses that included age as a factor. Fish for which the field gender identification could not be verified histologically (including four fish of the targeted species) were also excluded from analyses that included gender as a factor. Biomarker data for carp and bass were summarized and are presented in more detail than other species. 
All results for analytes in composite samples were converted to, reported as, and analyzed statistically as ww concentrations. A value of one-half the LOD was substituted for censored values in all statistical analyses and graphs. Concentrations of many analytes in composite samples were $<\mathrm{LOD}$, which limited the extent and rigor of the statistical analyses that could be performed. Geographic differences in concentrations of $p, p^{\prime}$-DDE, As, $\mathrm{Hg}$, Se, and $\mathrm{Zn}$ were examined statistically using ANOVA, as were temporal differences by combining the results of our study with 1970-1986 NCBP data from Stations 16, 63, 64, and 65 (Schmitt et al., 1999b). Because concentrations of total $\mathrm{Hg}$ in predatory fish increase with size, age, or both (see for example Lange et al., 1993; Wiener et al., 2002), temporal and geographic differences in logtransformed length-adjusted $(\mathrm{HgL})$ and weightadjusted $(\mathrm{HgW})$ concentrations computed as described by Brumbaugh et al. (2001) were also tested by ANOVA. A nominal $\alpha$-level of 0.05 was used in all statistical tests unless otherwise indicated. Details of the statistical procedures are given by Schmitt et al. (2004).

\section{Results}

\subsection{Lipid and moisture content (data not shown)}

Most composite samples were 1-5\% lipid, but lipid content varied among sites and species. Carp samples generally contained $2-7 \%$ lipid, but those from Stations 513 and 514 were 8.6-10.6\%. Lipid in channel catfish from Station 516 was $2.2-4.5 \%$, but at Station 511 it was $9.1-13.6 \%$. The blue catfish sample from Station 515 was also 10\% lipid. Conversely, lipid in striped bass from Station 63 was only $0.2 \%$. Most samples contained $70-78 \%$ water, but moisture content ranged from $65.3 \%$ in a carp sample from Station 514 to $79.8 \%$ in the striped bass from Station 63. Except for the striped bass, lipid and moisture values were typical for these taxa.

\subsection{Exposure indicators}

\subsubsection{Elemental contaminants}

Arsenic concentrations were $>$ LOD $(\sim 0.05 \mu \mathrm{g} / \mathrm{g}$ ww) in 38 samples (81\%) representing all ten stations. The greatest concentrations $(0.30-0.55 \mu \mathrm{g} /$ g) were in carp and white bass from Stations 65 and 514 , in the middle RGB; and in bass from Station 512, in the lower RGB (Fig. 2; Table 2). Elsewhere, concentrations in carp, bass channel catfish, and white bass were lower and generally similar (0.13$0.15 \mu \mathrm{g} / \mathrm{g}$ ), but were all $<$ LOD in northern pike from Station 64 (Fig. 2). Differences among stations were statistically significant in carp and bass, but not in blue or channel catfish (henceforth catfish) or in striped or white bass (Morone sp.; Table 2). Concentrations of As in 1997 differed significantly from historical levels in at least one taxon at Stations 63 , 64, and 65 , but there was only one obvious temporal trend-declining concentrations in Morone sp. at Station 65 (Table 3).

Selenium was detected in all samples at concentrations of $0.17-1.87 \mu \mathrm{g} / \mathrm{g}$, the latter in white bass from Station 65 (Fig. 2). Concentrations $\geq 0.5 \mu \mathrm{g} / \mathrm{g}$ were present in at least one sample from all stations, and all samples from Station 514 and two from each of Stations 515 and 65 contained $>1.0 \mu \mathrm{g} / \mathrm{g}$ (Fig. 2). Differences among or between stations were statistically significant in all taxa except catfish (Table 2), but the only statistically significant temporal difference was a downward trend in Morone sp. at Station 65 (Table 3).

Total $\mathrm{Hg}$ concentrations were $>$ LOD $(\sim 0.02 \mu \mathrm{g} / \mathrm{g}$ ww) in 45 of 47 samples (96\%) representing all 10 stations, and were generally greater in piscivores than in benthivores (Fig. 2). Greatest concentrations $(0.25 \mu \mathrm{g} / \mathrm{g})$ were in largemouth bass from Stations 63 and 514 and striped bass from Station 63; those in smallmouth bass and carp from Station 63 were lower $(<0.20 \mu \mathrm{g} / \mathrm{g}$; Fig. 2). Differences among stations were statistically significant only in Morone sp. and carp, however (Table 2). Total $\mathrm{Hg}$ and $\mathrm{HgL}$ concentrations in Morone sp. were significantly greater at Station 63 than at Station 65 , but $\mathrm{HgW}$ differences were only significant in carp (Fig. 2; Table 2). Temporal $\mathrm{Hg}$ differences were significant in some taxa, and these differences were consistent for total $\mathrm{Hg}, \mathrm{HgL}$, and $\mathrm{HgW}$ (Table 3). Overall, $\mathrm{Hg}$ concentrations in bass at Station 63, Morone sp. at Stations 63 and 65, and carp at Station 64 differed significantly among years, but there were no clearly evident temporal trends at any of these sites (Table 3). 

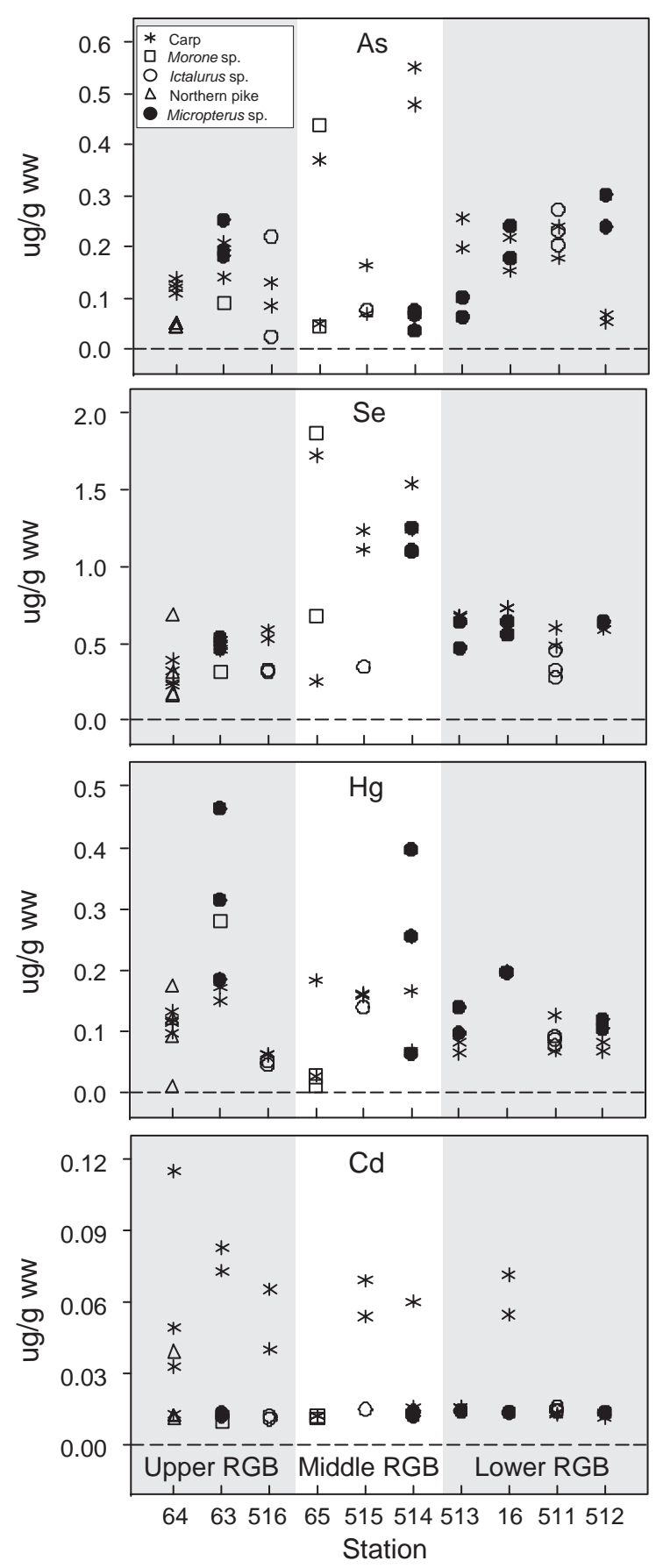

Fig. 2. Concentrations of arsenic (As), selenium (Se), and mercury $(\mathrm{Hg})$ in composite samples of whole fish, by sub-basin, station, and taxon. (Note: censored values are plotted as $50 \%$ of LOD.) Micropterus sp., largemouth or smallmouth bass; Morone sp., white or striped bass; Ictalurus sp., blue or channel catfish.
Lead concentrations were $>$ LOD $(\sim 0.03 \mu \mathrm{g} / \mathrm{g} \mathrm{ww})$ in 29 of 47 samples $(62 \%)$ representing eight stations; of the 29, $16(55 \%)$ were carp. Measured $\mathrm{Pb}$ concentrations ranged from 0.04 to $0.83 \mu \mathrm{g} / \mathrm{g}$, the latter in female bass from Station 16 (data not shown). In addition to the bass from Station 16, concentrations were generally greatest in carp from Stations 511, 512, and 513 and channel catfish from Station 511. Mean concentrations were lowest at Stations 63, 65 and 515 (Table 2), and most 1997 concentrations were lower than in the past (Table 3). The exception was Station 16, where the 1997 concentrations in bass were greater than in all previous collections (Table 3). Lead was not analyzed statistically because of the many censored values.

Cadmium concentrations were uniformly low; they exceed the LOD $(\sim 0.02 \mu \mathrm{g} / \mathrm{g}$ ww) in only 13 of 47 samples (28\%) and were also not analyzed statistically because of the many censored values. Of the 13 measured concentrations, 12 were in carp from six stations (Fig. 2). The maximum concentration was $0.12 \mu \mathrm{g} / \mathrm{g}$, in a carp from Station 64 (Fig. 2). Mean concentrations in carp were $\geq 0.05 \mu \mathrm{g} / \mathrm{g}$ only at Stations 16, 63, 515, and 516 (Table 2). Concentrations in all other taxa were lower. Temporal changes were not evident in any taxa (Table 3).

Zinc was detected in all samples and was generally greater in carp and northern pike than in other taxa (data not shown). Zinc concentrations ranged from 11.1 to $83.6 \mu \mathrm{g} / \mathrm{g}$, the latter in carp from Station 511 . Differences among stations were statistically significant only in carp; concentrations were significantly greater at Stations 16, 63, 511, and 515 than elsewhere (Table 2). The only significant temporal change was in white bass at Station 65, where concentrations were about two-fold greater in 1997 than in all previous collections (Table 3 ).

\subsubsection{Organochlorine pesticides}

Residues of comparatively few compounds [dichlorodiphenyltrichloroethane (DDT) and its metabolites, chlordane components, dieldrin, and toxaphene)] were detected in fish. These were found primarily at lower RGB sites (Fig. 3).

Concentrations of $p, p^{\prime}$-DDT, the active ingredient in commercial DDT, were $<$ LOD $(0.01 \mu \mathrm{g} / \mathrm{g})$ in all 1997 samples (data not shown). In contrast, residues of $p, p^{\prime}$-DDE, the most persistent metabolite of $p, p^{\prime}$ - 
Table 2

Least-squares geometric mean concentrations ${ }^{\mathrm{a}}$ (all wet-weight) of $p, p^{\prime}$-DDE (DDE), total mercury (Hg), length-adjusted total Hg (HgL), weight-adjusted total $\mathrm{Hg}(\mathrm{HgW})$, arsenic $(\mathrm{As})$, cadmium $(\mathrm{Cd})^{\mathrm{b}}$, lead $(\mathrm{Pb})^{\mathrm{b}}$, zinc $(\mathrm{Zn})$, and selenium $(\mathrm{Se})$ in composite samples of whole fish

\begin{tabular}{|c|c|c|c|c|c|c|c|c|c|}
\hline $\begin{array}{l}\text { Taxon, } \\
\text { station }(n)\end{array}$ & $\begin{array}{l}\text { DDE } \\
(\mu \mathrm{g} / \mathrm{g})\end{array}$ & $\begin{array}{l}\mathrm{Hg} \\
(\mu \mathrm{g} / \mathrm{g})\end{array}$ & $\begin{array}{l}\mathrm{HgL} \\
(\mu \mathrm{g} / \mathrm{g} / \mathrm{m})\end{array}$ & $\begin{array}{l}\mathrm{HgW} \\
(\mu \mathrm{g} / \mathrm{g} / \mathrm{kg})\end{array}$ & $\begin{array}{l}\text { As } \\
(\mu \mathrm{g} / \mathrm{g})\end{array}$ & $\begin{array}{l}\mathrm{Cd} \\
(\mu \mathrm{g} / \mathrm{g})\end{array}$ & $\begin{array}{l}\mathrm{Pb} \\
(\mu \mathrm{g} / \mathrm{g})\end{array}$ & $\begin{array}{l}\mathrm{Zn} \\
(\mu \mathrm{g} / \mathrm{g})\end{array}$ & $\begin{array}{l}\text { Se } \\
(\mu \mathrm{g} / \mathrm{g})\end{array}$ \\
\hline \multicolumn{10}{|l|}{ Carp (22) } \\
\hline $16(2)$ & $0.39 \mathrm{a}$ & $0.20 \mathrm{a}$ & $0.34 \mathrm{a}$ & $0.07 \mathrm{ab}$ & $0.18 \mathrm{abc}$ & 0.06 & 0.09 & $67.7 \mathrm{a}$ & $0.73 \mathrm{abc}$ \\
\hline $63(2)$ & $0.02 b$ & $0.16 \mathrm{a}$ & $0.34 \mathrm{a}$ & $0.13 \mathrm{a}$ & $0.17 \mathrm{abc}$ & 0.08 & 0.07 & $69.1 \mathrm{a}$ & $0.47 \mathrm{ad}$ \\
\hline $64(4)$ & $0.05 \mathrm{c}$ & $0.12 \mathrm{a}$ & $0.24 \mathrm{a}$ & $0.07 \mathrm{ab}$ & $0.12 b c$ & 0.04 & 0.10 & $62.6 \mathrm{a}$ & $0.29 \mathrm{~d}$ \\
\hline $65(2)$ & $0.02 b$ & $0.07 \mathrm{a}$ & $0.21 \mathrm{a}$ & $0.13 \mathrm{a}$ & $0.14 \mathrm{abc}$ & 0.01 & 0.04 & $32.3 b$ & $0.66 \mathrm{abc}$ \\
\hline $511(2)$ & $0.52 \mathrm{a}$ & $0.09 \mathrm{a}$ & $0.19 \mathrm{a}$ & $0.06 a b c$ & $0.21 \mathrm{abc}$ & 0.01 & 0.24 & $67.0 \mathrm{a}$ & $0.54 \mathrm{ac}$ \\
\hline $512(2)$ & $0.07 \mathrm{c}$ & $0.08 \mathrm{a}$ & $0.14 \mathrm{a}$ & $0.04 \mathrm{abc}$ & $0.06 \mathrm{~b}$ & 0.01 & 0.15 & $61.5 \mathrm{ab}$ & $0.61 \mathrm{ab}$ \\
\hline $513(2)$ & $0.21 \mathrm{ad}$ & $0.07 \mathrm{a}$ & $0.11 \mathrm{a}$ & $0.02 \mathrm{c}$ & $0.23 \mathrm{ac}$ & 0.02 & 0.15 & $46.8 \mathrm{ab}$ & $0.68 \mathrm{abc}$ \\
\hline $514(2)$ & $0.09 \mathrm{~d}$ & $0.11 \mathrm{a}$ & $0.17 \mathrm{a}$ & $0.03 \mathrm{bc}$ & $0.51 \mathrm{a}$ & 0.03 & 0.10 & $48.8 \mathrm{ab}$ & $1.38 \mathrm{~b}$ \\
\hline $515(2)$ & $0.15 \mathrm{~d}$ & $0.16 a$ & $0.25 \mathrm{a}$ & $0.04 \mathrm{abc}$ & $0.11 b c$ & 0.06 & 0.04 & $69.7 \mathrm{a}$ & $1.17 \mathrm{bc}$ \\
\hline $516(2)$ & $0.29 \mathrm{ad}$ & $0.06 \mathrm{a}$ & $0.11 \mathrm{a}$ & $0.03 \mathrm{bc}$ & $0.11 b c$ & 0.05 & 0.11 & $56.9 \mathrm{ab}$ & $0.56 \mathrm{ac}$ \\
\hline \multicolumn{10}{|c|}{ Ictalurus sp. (6) } \\
\hline $511(3)$ & $1.43 \mathrm{a}$ & $0.09 \mathrm{a}$ & $0.22 \mathrm{a}$ & $0.14 \mathrm{a}$ & $0.24 \mathrm{a}$ & 0.02 & 0.18 & $13.8 \mathrm{a}$ & $0.35 \mathrm{a}$ \\
\hline $515(1)$ & $0.10 \mathrm{~b}$ & $0.14 \mathrm{a}$ & $0.37 \mathrm{a}$ & $0.16 \mathrm{a}$ & $0.08 \mathrm{a}$ & 0.02 & 0.05 & $16.6 \mathrm{a}$ & $0.35 \mathrm{a}$ \\
\hline $516(2)$ & $0.11 \mathrm{~b}$ & $0.05 \mathrm{a}$ & $0.12 \mathrm{a}$ & $0.08 \mathrm{a}$ & $0.07 \mathrm{a}$ & 0.01 & 0.04 & $21.3 \mathrm{a}$ & $0.32 \mathrm{a}$ \\
\hline \multicolumn{10}{|c|}{ Micropterus sp. (12) } \\
\hline $16(2)$ & $0.38 \mathrm{a}$ & $0.20 \mathrm{a}$ & $0.52 \mathrm{a}$ & $0.24 \mathrm{a}$ & $0.21 \mathrm{ab}$ & 0.01 & 0.47 & $13.2 \mathrm{a}$ & $0.60 \mathrm{ab}$ \\
\hline $63(3)$ & $<0.01 \mathrm{~b}$ & $0.30 \mathrm{a}$ & $0.79 \mathrm{a}$ & $0.37 \mathrm{a}$ & $0.21 \mathrm{ab}$ & 0.01 & 0.04 & $15.3 \mathrm{a}$ & $0.51 \mathrm{a}$ \\
\hline $512(2)$ & $0.09 \mathrm{~cd}$ & $0.11 \mathrm{a}$ & $0.38 \mathrm{a}$ & $0.25 \mathrm{a}$ & $0.27 \mathrm{~b}$ & 0.01 & 0.04 & $15.8 \mathrm{a}$ & $0.64 \mathrm{ab}$ \\
\hline $513(2)$ & $0.15 \mathrm{~d}$ & $0.12 \mathrm{a}$ & $0.31 \mathrm{a}$ & $0.13 \mathrm{a}$ & $0.08 \mathrm{ab}$ & 0.01 & 0.08 & $13.8 \mathrm{a}$ & $0.55 \mathrm{ab}$ \\
\hline $514(3)$ & $0.05 \mathrm{c}$ & $0.19 \mathrm{a}$ & $0.62 \mathrm{a}$ & $0.32 \mathrm{a}$ & $0.06 \mathrm{a}$ & 0.01 & 0.03 & $12.7 \mathrm{a}$ & $1.15 \mathrm{c}$ \\
\hline \multicolumn{10}{|c|}{ Morone sp. (3) } \\
\hline $63(1)$ & $<0.01 \mathrm{a}$ & $0.28 \mathrm{a}$ & $0.39 \mathrm{a}$ & $0.12 \mathrm{a}$ & $0.09 \mathrm{a}$ & 0.01 & 0.03 & $19.2 \mathrm{a}$ & $0.32 \mathrm{a}$ \\
\hline $65(2)$ & $0.03 b$ & $0.02 b$ & $0.07 \mathrm{~b}$ & $0.10 \mathrm{a}$ & $0.14 \mathrm{a}$ & 0.01 & 0.04 & $31.5 \mathrm{a}$ & $1.13 \mathrm{~b}$ \\
\hline \multicolumn{10}{|c|}{ Northern pike (4) } \\
\hline $64(4)$ & 0.06 & 0.07 & 0.14 & 0.06 & 0.05 & 0.02 & 0.06 & 41.7 & 0.29 \\
\hline \multicolumn{10}{|l|}{$A N O V A$} \\
\hline$F$ & $41.67 * *$ & $5.44 * *$ & $5.97 * *$ & $7.10 * *$ & $4.21 * *$ & $\mathrm{ND}^{\mathrm{b}}$ & 5.79 & $\mathrm{ND}^{\mathrm{b}}$ & $7.03 * *$ \\
\hline$d f$ & 67,45 & 61,42 & 61,42 & 61,42 & 54,40 & $\mathrm{ND}^{\mathrm{b}}$ & 54,40 & $\mathrm{ND}^{\mathrm{b}}$ & 48,23 \\
\hline
\end{tabular}

Within each taxon-station group, means followed by the same letter are not significantly different $(P<0.01$, Fisher's protected LSD). Also shown are ANOVA $F$-values, degrees-of-freedom $(d f)$, and statistical significance $(* * P \leq 0.01)$.

a Censored values were represented by $50 \%$ of the LOD in all computations.

${ }^{\mathrm{b}} \mathrm{Cd}$ and $\mathrm{Pb}$ not tested statistically due to large numbers of censored (i.e., $<\mathrm{LOD}$ ) values.

DDT, were widespread; they were detected $(>0.01 \mu \mathrm{g} /$ g) in 43 of the 47 samples analyzed $(91 \%)$ and in at least one sample from all stations (Fig. 3). The greatest DDT concentrations (primarily as $p, p^{\prime}-$ DDE) were from Stations 16 and 511, in the lower RGB (Fig. 3, Table 2). Individual samples containing comparatively high concentrations of $p, p^{\prime}$-DDE $(\geq 0.5 \mu \mathrm{g} / \mathrm{g})$ included carp from Station $16(0.50 \mu \mathrm{g} /$ g) and carp and channel catfish from Station 511 $\left(0.67-1.60 \mu \mathrm{g} / \mathrm{g}\right.$; Fig. 2). Concentrations of $p, p^{\prime}-$
DDE differed significantly among stations in all taxa and were greatest in fish from lower RGB sites (Fig. 3, Table 2). The exception was Station 512, where concentrations were comparatively low. In addition to $p, p^{\prime}$-DDE, residues of $p, p^{\prime}$-DDD (TDE) were detected $(>0.01 \mu \mathrm{g} / \mathrm{g})$ in 23 of 47 samples representing six stations, but concentrations were uniformly low $(\leq 0.084 \mu \mathrm{g} / \mathrm{g}$; data not shown). Traces of $o, p^{\prime}$-DDT $(0.03-0.06 \mu \mathrm{g} / \mathrm{g})$ and $o, p^{\prime}-$ DDD (TDE; 0.02-0.03 $\mu \mathrm{g} / \mathrm{g}$ ) were present exclu- 
Table 3

Least-squares geometric mean concentrations ${ }^{\mathrm{a}}$ (all wet-weight) of $p, p^{\prime}$-DDE, total mercury ( $\mathrm{Hg}$ ), length-adjusted total $\mathrm{Hg}$ (HgL), weightadjusted total $\mathrm{Hg}(\mathrm{HgW})$, arsenic (As), cadmium $(\mathrm{Cd})^{\mathrm{b}}$, lead $(\mathrm{Pb})^{\mathrm{b}}$, zinc $(\mathrm{Zn})$, and selenium (Se) in fish collected from 1970 to 1997 at National Contaminant Biomonitoring Program (NCBP) stations (Schmitt et al., 1999b) in the Rio Grande basin

\begin{tabular}{lccllllllll}
\hline $\begin{array}{l}\text { Station, location, } \\
\text { taxon }\end{array}$ & Year & $\begin{array}{l}\mathrm{DDE} \\
(\mu \mathrm{g} / \mathrm{g})\end{array}$ & $\begin{array}{l}\mathrm{Hg} \\
(\mu \mathrm{g} / \mathrm{g})\end{array}$ & $\begin{array}{l}\mathrm{HgL} \\
(\mu \mathrm{g} / \mathrm{g} / \mathrm{m})\end{array}$ & $\begin{array}{l}\mathrm{HgW} \\
(\mu \mathrm{g} / \mathrm{g} / \mathrm{kg})\end{array}$ & $\begin{array}{l}\mathrm{As} \\
(\mu \mathrm{g} / \mathrm{g})\end{array}$ & $\begin{array}{l}\mathrm{Cd} \\
(\mu \mathrm{g} / \mathrm{g})\end{array}$ & $\begin{array}{l}\mathrm{l} \mathrm{Pb} \\
(\mu \mathrm{g} / \mathrm{g})\end{array}$ & $\begin{array}{l}\mathrm{Zn} \\
(\mu \mathrm{g} / \mathrm{g})\end{array}$ & $\begin{array}{l}\mathrm{Se} \\
(\mu \mathrm{g} / \mathrm{g})\end{array}$ \\
\hline Station 16, Rio Grande at Mission, TX & & & & & & & & \\
Carp & 1981 & 0.44 & $0.05^{*}$ & 0.11 & 0.04 & 0.15 & 0.03 & 0.10 & 41.0 & 0.40 \\
& 1997 & 0.39 & 0.20 & 0.34 & 0.07 & 0.18 & 0.06 & 0.09 & 67.7 & 0.73 \\
Micropterus sp. & 1980 & $2.57^{* *}$ & 0.24 & 0.64 & 0.29 & 0.06 & 0.01 & 0.10 & 12.7 & 0.57 \\
& 1984 & $2.09^{* *}$ & 0.18 & 0.50 & 0.26 & 0.11 & 0.01 & 0.07 & 13.2 & 0.44 \\
& 1986 & $2.73^{* *}$ & 0.26 & 0.57 & 0.17 & 0.17 & $<0.01$ & 0.12 & 11.6 & 0.49 \\
& 1997 & 0.38 & 0.20 & 0.52 & 0.24 & 0.21 & 0.01 & 0.47 & 13.2 & 0.60
\end{tabular}

Station 63, Rio Grande at Elephant Butte Reservoir, NM

\begin{tabular}{|c|c|c|c|c|c|c|c|c|c|c|}
\hline \multirow[t]{8}{*}{ Carp } & 1972 & $0.11 * *$ & 0.16 & 0.51 & 0.35 & 0.13 & 0.03 & 0.17 & ND & $0.64 *$ \\
\hline & 1973 & $0.11 * *$ & 0.11 & 0.30 & 0.20 & 0.05 & 0.03 & 0.05 & ND & 0.23 \\
\hline & 1974 & $0.09 * *$ & ND & ND & ND & ND & ND & ND & ND & ND \\
\hline & 1978 & $0.07 * *$ & 0.26 & 0.67 & $0.38 *$ & $0.04 *$ & 0.13 & 0.28 & 84.7 & 0.50 \\
\hline & 1980 & $0.04 *$ & 0.16 & 0.39 & 0.20 & 0.12 & 0.08 & 0.30 & 68.9 & 0.41 \\
\hline & 1984 & 0.01 & 0.11 & 0.24 & 0.06 & 0.08 & 0.07 & 0.25 & 51.8 & 0.29 \\
\hline & 1986 & 0.01 & 0.13 & 0.31 & 0.13 & 0.14 & 0.04 & 0.18 & 52.2 & 0.34 \\
\hline & 1997 & 0.02 & 0.16 & 0.34 & 0.13 & 0.17 & 0.08 & 0.07 & 69.1 & 0.47 \\
\hline \multirow[t]{8}{*}{ Micropterus sp. } & 1970 & $0.23 * *$ & 0.52 & 1.10 & 0.25 & ND & ND & ND & ND & ND \\
\hline & 1971 & $0.15 * *$ & 0.60 & 1.41 & 0.48 & 0.13 & 0.03 & 0.05 & ND & ND \\
\hline & 1972 & $0.09 * *$ & 0.28 & 0.73 & 0.31 & 0.27 & 0.03 & 0.05 & ND & 0.66 \\
\hline & 1973 & $0.05^{* *}$ & $0.05^{* *}$ & $0.16^{* *}$ & $0.08^{* *}$ & 0.17 & 0.03 & 0.05 & ND & $0.20 *$ \\
\hline & 1978 & $0.10 * *$ & 0.65 & 1.69 & 0.57 & 0.16 & 0.01 & 0.18 & 16.3 & 0.56 \\
\hline & 1980 & $0.03 * *$ & 0.17 & 0.50 & 0.29 & 0.27 & 0.01 & 0.10 & 12.9 & 0.41 \\
\hline & 1984 & 0.01 & 0.12 & 0.45 & 0.44 & 0.27 & $<0.01$ & 0.01 & 13.8 & 0.36 \\
\hline & 1997 & $<0.01$ & 0.30 & 0.79 & 0.37 & 0.21 & 0.01 & 0.04 & 15.3 & 0.51 \\
\hline \multirow[t]{2}{*}{ Morone sp. } & 1971 & $0.17 * *$ & 0.63 & 1.55 & $0.69 * *$ & 0.18 & 0.03 & 0.05 & ND & ND \\
\hline & 1997 & $<0.01$ & 0.28 & 0.39 & 0.12 & 0.09 & 0.01 & 0.03 & ND & ND \\
\hline \multicolumn{11}{|c|}{ Station 64, Rio Grande at Alamosa, NM } \\
\hline \multirow[t]{4}{*}{ Carp } & 1970 & $0.23 * *$ & $0.03 *$ & $0.06 *$ & $0.02 *$ & ND & ND & ND & ND & ND \\
\hline & 1971 & $0.11 * *$ & 0.08 & 0.30 & $0.34 * *$ & $0.04 *$ & 0.03 & 0.19 & ND & ND \\
\hline & 1972 & $0.24 * *$ & 0.05 & 0.13 & 0.05 & 0.08 & 0.03 & 4.65 & ND & 0.38 \\
\hline & 1997 & 0.05 & 0.12 & 0.24 & 0.07 & 0.12 & 0.04 & 0.10 & ND & 0.29 \\
\hline \multicolumn{11}{|c|}{ Station 65, Pecos River at Red Bluff Lake, TX } \\
\hline \multirow[t]{2}{*}{ Carp } & 1986 & $0.08 * *$ & 0.04 & 0.13 & 0.09 & $0.01 * *$ & 0.01 & 0.28 & 52.9 & 0.63 \\
\hline & 1997 & 0.02 & 0.07 & 0.21 & 0.13 & 0.14 & 0.01 & 0.04 & 32.3 & 0.66 \\
\hline \multirow[t]{6}{*}{ Morone sp. } & 1974 & $0.47 * *$ & ND & ND & ND & ND & ND & ND & ND & ND \\
\hline & 1978 & $0.17 * *$ & $0.12 * *$ & $0.33^{*}$ & 0.15 & $0.66^{*}$ & 0.01 & 0.10 & 20.1 & $3.05 *$ \\
\hline & 1980 & $0.14 * *$ & 0.06 & 0.14 & 0.05 & $0.60 *$ & 0.01 & 0.10 & $11.2 * *$ & 1.96 \\
\hline & 1984 & $0.08 *$ & 0.06 & 0.22 & 0.22 & 0.24 & $<0.01$ & 0.06 & $14.2 *$ & 1.50 \\
\hline & 1986 & $0.16^{* *}$ & $0.14 * *$ & $0.31^{*}$ & 0.09 & 0.32 & $<0.01$ & 0.14 & $13.0^{*}$ & 1.00 \\
\hline & 1997 & 0.03 & 0.02 & 0.07 & 0.10 & 0.14 & 0.01 & 0.04 & 31.5 & 1.13 \\
\hline ANOVA- $F$ & - & $41.67 * *$ & $5.44 * *$ & $5.97 * *$ & $7.10^{* *}$ & $4.21 * *$ & $\mathrm{ND}^{\mathrm{b}}$ & $\mathrm{ND}^{\mathrm{b}}$ & $13.45^{* *}$ & $7.03^{* *}$ \\
\hline$d f$ & - & 67,45 & 61,42 & 61,42 & 61,42 & 54,40 & $\mathrm{ND}^{\mathrm{b}}$ & $\mathrm{ND}^{\mathrm{b}}$ & 37,28 & 48,23 \\
\hline
\end{tabular}

Within each group of station-taxon means, values followed by asterisks $(*)$ differ significantly $(* 0.01<P \leq 0.05$; ** $P \leq 0.01$, Fisher's protected LSD) from 1997 means. Also shown are ANOVA $F$-values, degrees-of-freedom $(d f)$, and significance levels. ND, no data/not measured.

${ }^{a}$ Censored values (i.e., $<$ LOD) were represented by $50 \%$ of the LOD in all computations.

${ }^{\mathrm{b}} \mathrm{Cd}$ and $\mathrm{Pb}$ not tested statistically due to large numbers of censored values. 

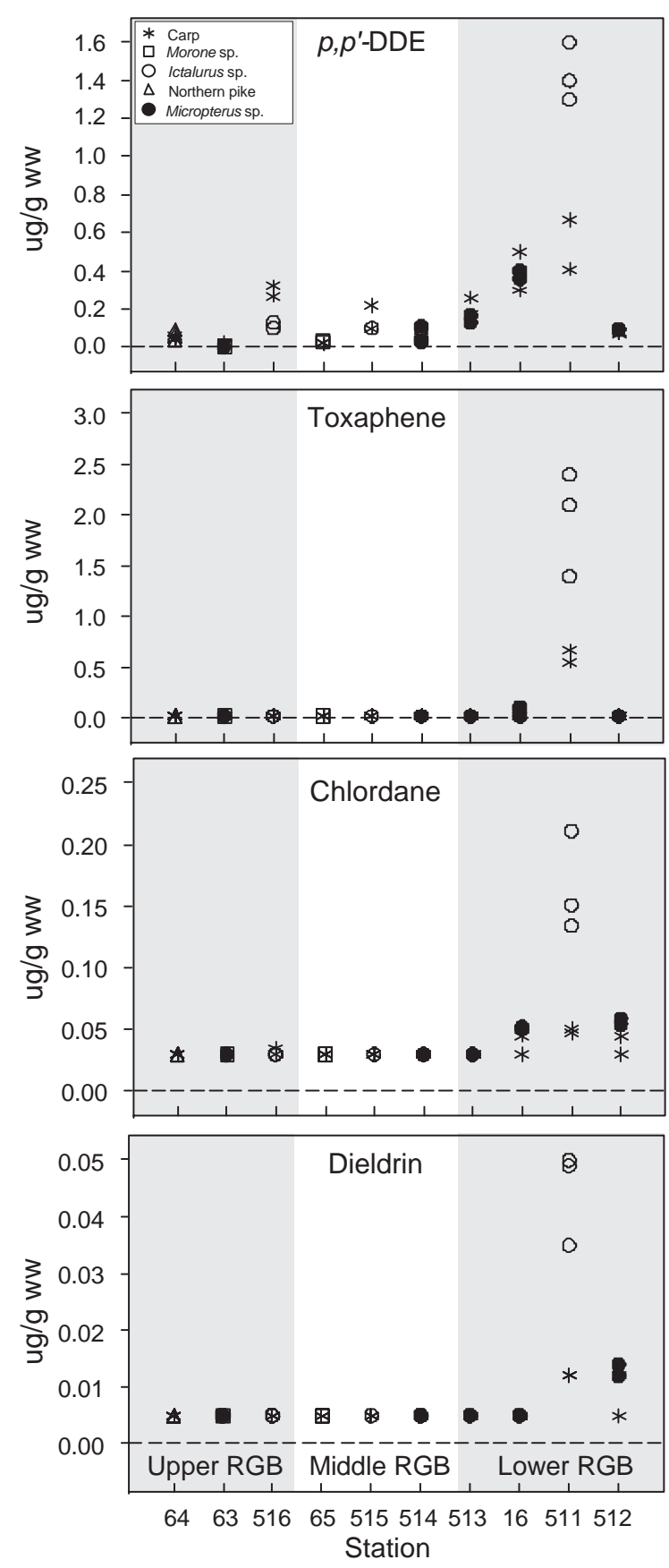

Fig. 3. Concentrations of $p, p$-DDE, total chlordanes (sum of $c i s$-and trans-chlordanes and nonachlors, oxychlordane, and heptachlor epoxide), dieldrin, and toxaphene in composite samples of whole fish, by sub-basin, station, and taxon. (Note: censored values are plotted as $50 \%$ of LOD.) Micropterus sp., largemouth or smallmouth bass; Morone sp., white or striped bass; Ictalurus sp., blue or channel catfish. sively in the three channel catfish samples from Station 511, but $o, p^{\prime}$-DDE residues were not detected $(<0.01 \mu \mathrm{g} / \mathrm{g})$ in any samples (data not shown). Concentrations of $p, p^{\prime}$-DDE declined significantly in at least one taxon at all four NCBP stations sampled in 1997 (Table 3).

Six chlordane-related compounds (cis- and transchlordanes and nonachlors; heptachlor epoxide; and oxychlordane) were measured. Only cis- and transchlordanes and nonachlors were detected and only in samples from Stations 16, 511, 512, and 516 (Fig. 3). Concentrations of oxychlordane and heptachlor epoxide were universally $<$ LOD $(0.01 \mu \mathrm{g} / \mathrm{g})$. Chlordane concentrations (sum of all components) were greatest in channel catfish from Station 511 (Fig. 3). Transnonachlor was the most frequently detected chlordane constituent; it was detected $(0.036-0.073 \mu \mathrm{g} / \mathrm{g})$ in 12 samples (26\%) representing four stations $(16,511$, 512, and 516). It was also the most abundant component except at Station 511, where cis-chlordane was most abundant and was detected $(0.017-0.073$ $\mu \mathrm{g} / \mathrm{g})$ in both channel catfish and carp. Traces $(<0.02$ $\mu \mathrm{g} / \mathrm{g}$ ) of cis-chlordane were also detected in bass from Station 512 and carp from Station 16. Trans-chlordane $(0.015-0.022 \mu \mathrm{g} / \mathrm{g})$ and cis-nonachlor $(0.019-$ $0.033 \mu \mathrm{g} / \mathrm{g}$ ) were detected only in channel catfish from Station 511.

Dieldrin was detected $(>0.01 \mu \mathrm{g} / \mathrm{g})$ in only $17 \%$ of the samples representing two sites (Fig. 3). Concentrations were greatest $(0.035-0.05 \mu \mathrm{g} / \mathrm{g})$ in channel catfish from Station 511. Both carp samples from Station 511 and three of the four samples from Station 512 (carp and largemouth bass) also contained traces of dieldrin $(<0.015 \mu \mathrm{g} / \mathrm{g})$.

Toxaphene was detected $(>0.05 \mu \mathrm{g} / \mathrm{g})$ in only six samples representing two lower RGB stations (16 and 511; Fig. 3). Concentrations at Station 16 were low $(<0.1 \mu \mathrm{g} / \mathrm{g})$ whereas those in fish from Station 511 were $0.56-2.40 \mu \mathrm{g} / \mathrm{g}$, with those in channel catfish greatest.

\subsubsection{Total PCBs and TCDD-EQ (data not shown)}

Total PCB concentrations were $<$ LOD $(0.05 \mu \mathrm{g} / \mathrm{g})$ in all 1997 samples. The H4IIE bioassay also detected relatively low dioxin-like activity; TCDDEQ was $\leq 6 \mathrm{pg} / \mathrm{g}$ in all samples, and was $\geq 4 \mathrm{pg} / \mathrm{g}$ in only three samples (one from Station 511, two from Station 512). 


\subsubsection{Hepatic ethoxyresorufin O-deethylase (EROD) activity}

In carp, statistically significant ANOVA models containing the factors station, gender, gonadal stage, and their interactions explained $54-56 \%$ of the total variation in EROD activity $\left(F_{28}, 163=7.53, P<0.01\right)$. Differences among stations were highly significant $\left(F_{9,172}=19.62, P<0.01\right)$ as were those between genders $\left(F_{1,172}=13.24, P<0.01\right)$; activity was greater in males than in females. Station-gender interaction was not significant $\left(F_{9,172}=1.00, P>0.05\right)$, indicating that most among-station differences were similar in both genders; EROD activity was elevated at Stations 511 and 512 (means $=8.70-32.6 \mathrm{pmol} / \mathrm{min} / \mathrm{mg}$ ) and lowest at Station $64(0.25-0.34 \mathrm{pmol} / \mathrm{min} / \mathrm{mg})$ in both genders (Table 4). However, EROD activity was also elevated relative to most sites in male carp from Station $516($ mean $=9.24 \mathrm{pmol} / \mathrm{min} / \mathrm{mg})$, but not in female carp (Table 4). Slightly elevated EROD rates $(>4 \mathrm{pmol} / \mathrm{min} / \mathrm{mg}$ ) characterized at least one carp from all sites. In addition, one carp from Station 65 and most from Stations 511 and 512 were $>10 \mathrm{pmol} / \mathrm{min} / \mathrm{mg}$.

In bass, ANOVA models containing the factors station, gender, gonadal stage, and their interactions were also statistically significant $\left(F_{15},{ }_{56}=4.91\right.$, $P<0.01)$ and explained $57 \%$ of the total variation in EROD activity. Of 30 male bass analyzed, only two had EROD rates $<20.0 \mathrm{pmol} / \mathrm{min} / \mathrm{mg}$ and most females were $>16.0 \mathrm{pmol} / \mathrm{min} / \mathrm{mg}$ (Table 4). Activity in bass was generally greatest at Stations 63 and 512 in both males and females; however, bass were not obtained at Stations 511 and 516, where elevated EROD rates were detected in carp, and only female bass were obtained at Station 63. All values in bass from Stations 63 and 512 were $>25.0 \mathrm{pmol} / \mathrm{min} / \mathrm{mg}$ (Table 4). In largemouth bass, differences among stations were highly significant $\left(F_{4}, 63=8.90\right.$, $P<0.01)$ as were those between genders $\left(F_{1,63}=\right.$ $4.85, P<0.05)$. As was true for carp, activity was significantly greater in males than in females and interactions between station and gender were not significant $\left(F_{4,63}=0.96, P>0.05\right)$, indicating that the geographic differences were consistent between genders. Activity was significantly lower in largemouth bass of both genders from Station 514 than from all other sites and was greatest at Station 512 (Table 4).

In addition to carp and bass, EROD activity was measured in 47 fish representing five other species (channel catfish, blue catfish, northern pike, white bass, and striped bass) from five of the 10 stations sampled (Table 4). In channel catfish, EROD rates differed significantly among stations $\left(F_{1,37}=9.46\right.$, $P<0.01)$ but not genders $\left(F_{2,37}=0.26, P>0.05\right)$. The interaction of station and gender was also not significant $\left(F_{2}, 37=2.33, P>0.05\right)$. Activity was several-fold greater in channel catfish from Station 511 than in either channel catfish from Station 516 or blue catfish from Station 515, and the channel catfish differences were consistent for males, females, and juveniles (Table 4).

\subsection{Fish health indicators}

\subsubsection{External lesions and health assessment index (HAI)}

Of the 368 fish examined, $28 \%$ had some type of external lesion, most of which were identified as eroded, frayed, or hemorrhagic fins. Lesion frequencies (all species combined) ranged from $2 \%$ at Station 65 to $75 \%$ at Station 515 (data not shown). Of the 207 carp examined, $29 \%$ had external lesions. Percentages for carp were lowest at Stations 16, 65, and 512 and highest at Stations 516 and 515. No carp from Station 65 had external lesions where occurrence was $90 \%$ at Station 515, which was 3-9 times greater than most other stations. Of the 75 bass examined, $27 \%$ had external lesions. Percentages for bass were lowest at Stations $16(1 \%)$ and $513(14 \%)$ but were $\geq 50 \%$ at Stations 512 and 514.

Most $(90 \%)$ of the HAI scores for carp were between 20 and 90, and all station means except for Station 65 were between 30 and 70; Station 65 scored lower than most (Fig. 4). Station means were $>60$ only at Stations 511 and 515, and except for Station 515, $80-100 \%$ of the carp from each station scored $<100$ (Fig. 4). For bass, most HAI scores $(90 \%)$ were between 10 and 110, but only the Station 512 mean was $<50$; means for Stations 514 and 63 were $>60$, and no individual bass from these sites scored $<40$. The greatest means for carp and bass also did not occur at the same stations, but bass were not collected at Stations 511 or 515 , where scores in carp were generally greatest. Statistically significant ANOVA models that included the factors station, gender, stage, and their interactions accounted for $28 \%$ of the total HAI variation (rank-transformed) in carp and $41 \%$ in 
Table 4

Geometric means ${ }^{\mathrm{a}}$ and ranges of hepatic ethoxyresorufin $O$-deethylase (EROD) activity (all in $\mathrm{pmol} / \mathrm{min} / \mathrm{mg}$ protein), by species, station, and gender

\begin{tabular}{|c|c|c|c|c|c|c|c|c|c|}
\hline \multirow{2}{*}{$\begin{array}{l}\text { Species } \\
\text { and station }\end{array}$} & \multicolumn{3}{|c|}{ Female } & \multicolumn{3}{|c|}{ Male } & \multicolumn{3}{|c|}{ Juvenile $^{\mathrm{b}}$} \\
\hline & $n$ & Range & Mean & $n$ & Range & Mean & $n$ & Range & Mean \\
\hline \multicolumn{10}{|l|}{ Carp $^{\mathrm{c}}$} \\
\hline 16 & 10 & $0.76-3.35$ & $1.31 \mathrm{a}$ & 10 & $0.72-4.47$ & $2.89 \mathrm{~b}$ & 0 & - & - \\
\hline 63 & 8 & $0.18-4.95$ & $1.81 \mathrm{a}$ & 9 & $0.18-27.2$ & $4.76 c$ & 0 & - & - \\
\hline 64 & 19 & $0.07-2.72$ & $0.25 b$ & 14 & $0.08-7.84$ & $0.34 \mathrm{a}$ & 1 & - & 0.07 \\
\hline 65 & 10 & $0.03-55.5$ & $1.90 \mathrm{a}$ & 10 & $0.03-7.38$ & $1.54 \mathrm{~b}$ & 0 & - & - \\
\hline 511 & 13 & $0.06-161$ & $16.8 \mathrm{c}$ & 7 & $20.1-54.2$ & $32.6 \mathrm{e}$ & 0 & - & - \\
\hline 512 & 6 & $3.19-15.1$ & $9.44 \mathrm{c}$ & 8 & $2.0-18.8$ & $8.70 \mathrm{df}$ & 0 & - & - \\
\hline 513 & 11 & $0.49-7.98$ & $1.80 \mathrm{a}$ & 9 & $2.58-6.88$ & $3.97 b c$ & 0 & - & - \\
\hline 514 & 10 & $0.12-3.42$ & $1.42 \mathrm{a}$ & 10 & $0.06-8.74$ & $3.46 \mathrm{bc}$ & 0 & - & - \\
\hline 515 & 6 & $0.02-2.98$ & $0.86 \mathrm{a}$ & 4 & $0.57-4.50$ & $2.13 \mathrm{bcd}$ & 0 & - & - \\
\hline 516 & 10 & $0.81-2.83$ & $1.49 \mathrm{a}$ & 8 & $2.26-28.0$ & $9.24 \mathrm{ef}$ & 0 & - & - \\
\hline \multicolumn{10}{|c|}{ Largemouth bass ${ }^{\mathrm{d}}$} \\
\hline $16^{\circ}$ & 11 & $9.09-58.5$ & $28.6 a b$ & 10 & $33.5-132$ & $54.8 \mathrm{a}$ & 0 & - & - \\
\hline 63 & 4 & $38.5-81.0$ & $57.7 \mathrm{c}$ & 0 & - & - & 2 & $62.9-130$ & 90.3 \\
\hline 512 & 7 & $28.1-107$ & $67.4 \mathrm{c}$ & 8 & $46.2-107$ & $75.9 \mathrm{a}$ & 1 & - & 111.2 \\
\hline 513 & 13 & $15.5-96.6$ & $37.2 b$ & 9 & $19.7-83.5$ & $50.6 \mathrm{a}$ & 0 & - & - \\
\hline 514 & 5 & $5.00-44.3$ & $21.3 \mathrm{a}$ & 2 & $13.0-22.3$ & $17.0 \mathrm{~b}$ & 0 & - & - \\
\hline \multicolumn{10}{|c|}{ Smallmouth bass } \\
\hline 63 & 2 & $79.6-147$ & 108 & 0 & - & - & 0 & - & - \\
\hline 514 & 0 & - & - & 1 & - & 52.6 & 0 & - & - \\
\hline \multicolumn{10}{|l|}{ Striped bass } \\
\hline 63 & 1 & - & $<0.1$ & 0 & - & - & 0 & - & - \\
\hline \multicolumn{10}{|l|}{ White bass } \\
\hline 65 & 18 & $2.50-22.4$ & 9.52 & 3 & $2.77-8.36$ & 4.34 & 0 & - & - \\
\hline \multicolumn{10}{|l|}{ Blue catfish } \\
\hline 515 & 3 & $7.19-17.2$ & 10.3 & 0 & - & - & 3 & $9.75-12.2$ & 11.0 \\
\hline \multicolumn{10}{|c|}{ Channel catfish ${ }^{\mathrm{e}}$} \\
\hline 511 & 8 & $17.9-71.7$ & $40.6 \mathrm{a}$ & 3 & $12.8-27.9$ & $20.3 \mathrm{a}$ & 9 & $18.9-111$ & $38.9 \mathrm{a}$ \\
\hline 516 & 11 & $6.12-23.1$ & $12.2 b$ & 2 & $15.8-19.5$ & $17.5 \mathrm{a}$ & 9 & $9.3-25.4$ & $14.1 \mathrm{a}$ \\
\hline \multicolumn{10}{|c|}{ Northern pike } \\
\hline 64 & 6 & $0.14-1.77$ & 0.52 & 10 & $0.18-2.30$ & 0.69 & 0 & - & - \\
\hline
\end{tabular}

Within each species-gender group for common carp, largemouth bass, and channel catfish, means followed by the same letter are not significantly different ( $P>0.05$, Fisher's protected LSD).

${ }^{\text {a }}$ Censored values (i.e., $<$ LOD) were represented by $50 \%$ of the limit-of-quantitation in the computation of geometric means.

b May include fish of undetermined gender from which no gonad sample was obtained.

c $\operatorname{ANOVA} F_{(19,172)}=10.68, P<0.01, R^{2}=0.54$.

d ANOVA $F_{(8,63)}=5.58, P<0.01, R^{2}=0.42$.

e ANOVA $F_{(5,32)}=9.46, P<0.01, R^{2}=0.60$.

bass. Differences among gonadal stages were statistically significant in both taxa, as was the interaction of station, stage, and gender in bass. However, after accounting for all other effects, differences among stations were not significant in carp and only approached significance in bass $\left(F_{4}, 56=2.01, P=\right.$ $0.11)$. Differences between genders were not significant in either carp or bass. 


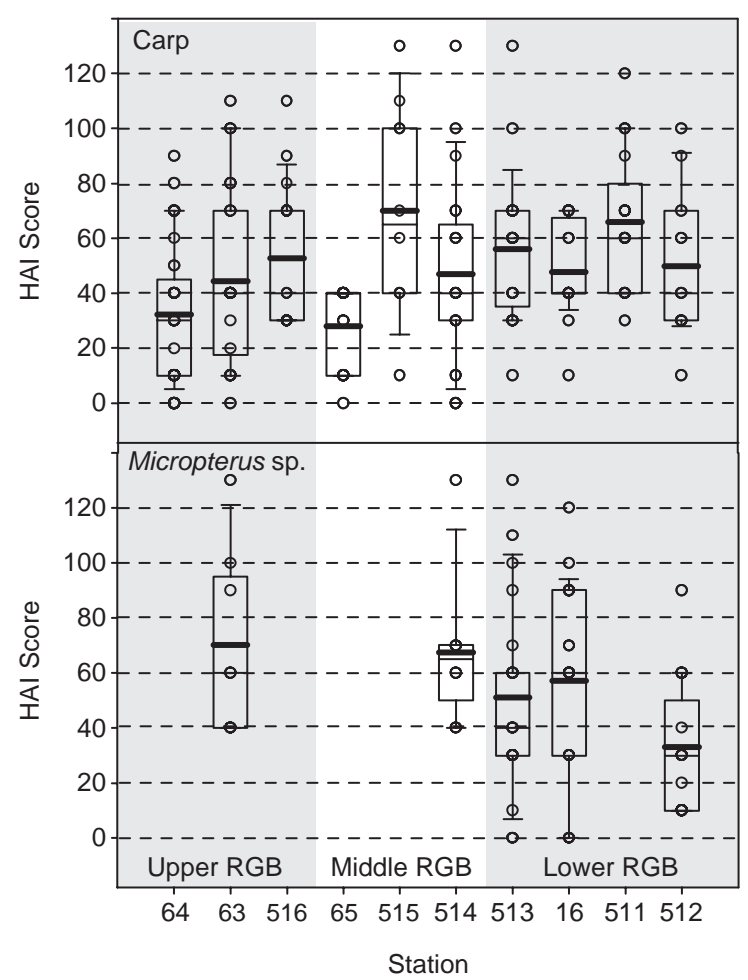

Fig. 4. Health assessment index (HAI) scores of carp and bass (Micropterus sp.), by sub-basin and station. Shown for each group are points representing individual fish and the mean (heavy horizontal line), median (light horizontal line), interquartile range (box), and the 10th and 90th percentiles (whiskers). Stations are ordered from upstream to downstream and are grouped by sub-basin.

Internal and external lesions contributing to elevated HAI ratings in carp and bass were examined histopathologically to determine their cause. Overall, most microscopic lesions were diagnosed as inflammatory and were usually associated with parasites. In bass, abnormal ratings at Station 63 were primarily due to frayed or marginate gills and abnormal livers; the latter were all associated with helminth parasites. Most of the abnormal ratings for Station 16 bass resulted from internal nodules, focal discolorations, or spots on the liver, kidney, and spleen, which were also determined to be parasite-induced. Many Station 513 bass kidneys were rated in the field as urolithic; however, histologically no calcifications were noted and these lesions were also determined to be parasiteinduced. In carp, high HAI scores at Station 515 were primarily due to external lesions; the only abnormal internal ratings were for tan livers, which were also noted at Stations 511, 513, and 516. Two Station 515 carp had unusual inflammatory reactions in the epidermis or hypodermis associated with refractile, crystalline material (Fig. 5A, B). In addition, two carp from each of Stations 516 and 514 had papillomas of unknown etiology on the body surface or oral cavity (Fig. 5C). Histologically, the tan livers were found to contain hepatocytes with increased vacuolization, and ceroid/lipofuscin deposits were often contained within or replacing hepatocytes. Ceroid-containing cells were also present within the blood vessel walls in the livers of many carp from these stations (Fig. 5D). Four of 14 carp from Station 512 had abnormal kidneys rated as granular. In these four fish (and in one not identified as granular in the field), and in contrast to the bass described previously, histopathological analysis revealed urolithiasis or nephrocalcinosis (Fig. 5E).

Among the changes detected during histopathological examination was an apparent proliferation of thyroid follicles in the posterior kidney of carp from some sites (Fig. 5F); these were not evident during gross examination. Carp kidneys from Stations 63, 64 and 516 contained few thyroid follicles and those that were present tended to be small whereas greater numbers and larger size characterized the follicles of carp from stations further downstream, and the kidneys of one carp from Station 514 contained a large area of abnormal-appearing thyroid follicles. Further analyses are being conducted to determine thyroid hormone status and to provide additional quantitative and qualitative information on the kidney thyroid follicles.

\subsubsection{Condition factor and organosomatic indices (data not shown)}

Condition factor differences in carp were not statistically significant; an ANOVA model that included the factors station, gender, gonadal stage, and their interactions accounted for only $9 \%$ of the total variation $\left(F_{28}, 176=0.67, P>0.05\right)$. Station means ranged from 1.2 at Stations 63 and 512 to 1.6 at Stations 513 and 514, and $90 \%$ of the individual values were between 1.1 and 1.8. In contrast to carp, ANOVA was highly significant $\left(F_{15,56}=12.94, P<0.01\right)$ and explained $78 \%$ of $C F$ variation in bass; among-station differences were also significant $\left(F_{4}, 56=3.78\right.$, $P<0.01)$. Station means ranged from 1.4 at Stations 


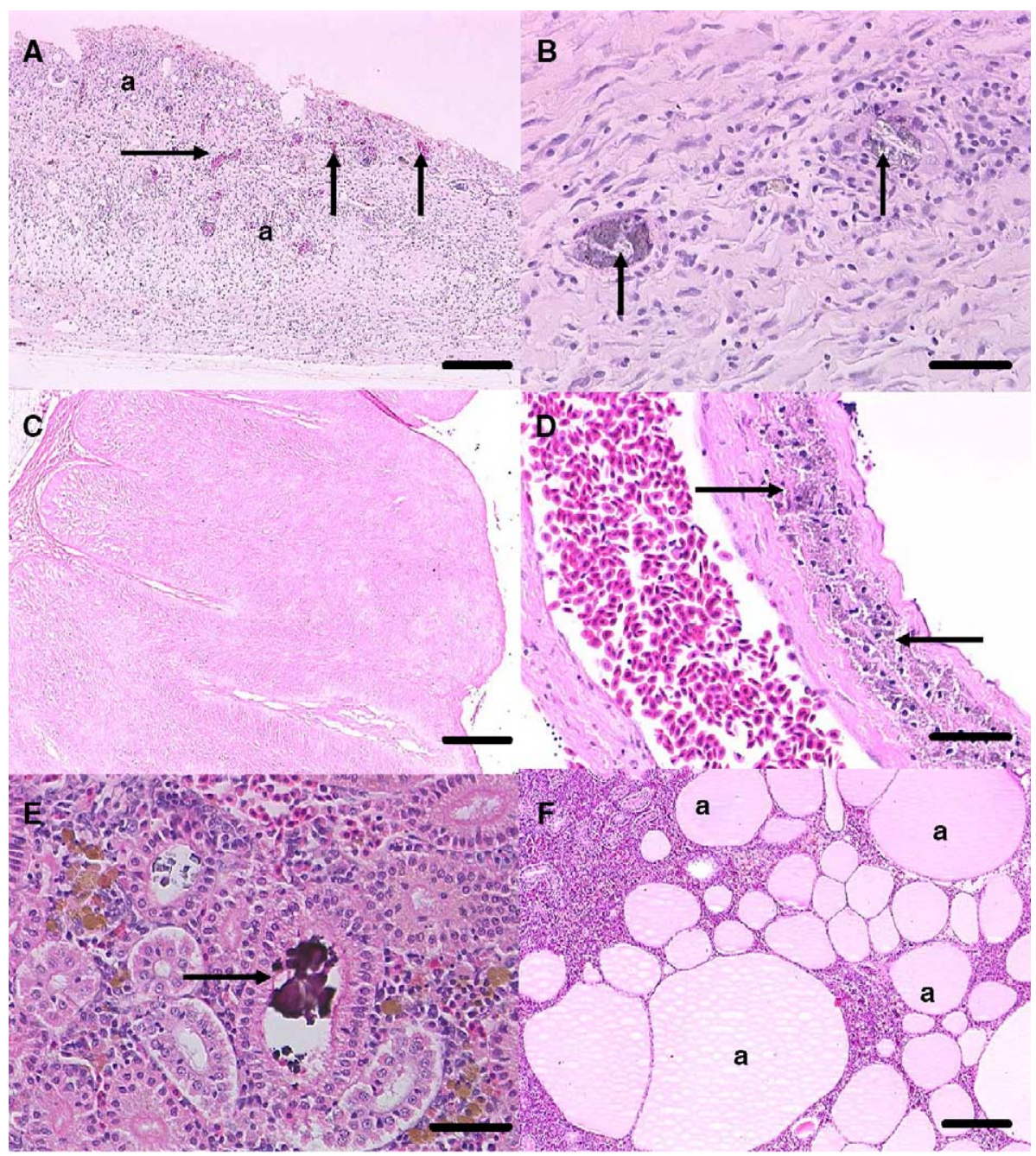

Fig. 5. A. Histologic appearance of external gross skin lesions observed in carp from station 515 in the Rio Grande basin. Inflammatory responses included chronic inflammation (a) and congestion (thin arrows) throughout a thickened epidermis and dermis. Scale bar=200 $\mu \mathrm{m}$. B. Higher magnification of a portion of (A) illustrating the refractile, crystalline material that appears to elicit this inflammatory response (arrows). Scale bar $=50 \mu \mathrm{m}$. C. Papilloma in the skin of carp from Station 514. Scale bar $=200 \mu \mathrm{m}$. D. Pigmented cell accumulations within the hepatic blood vessel walls (arrows) typical of carp from Stations 515, 511, 513 and 516. Scale bar $=50 \mu \mathrm{m}$. E. Nephrocalcinosis or mineral deposition within tubules (arrow) was observed at Station 512. Scale bar $=50 \mu \mathrm{m}$. F. Posterior kidney tissue of carp from station 513 illustrating accumulations of enlarged thyroid follicles (a). Scale bar $=200 \mu \mathrm{m}$. H and E stain.

16 and 63 to 1.8 at Station 514 , and $90 \%$ of the bass examined had CF values of 1.2-2.0.

Hepatosomatic index differences (bass only) were not statistically significant; an ANOVA model that included the factors station and gender and their interactions explained only $12 \%$ of the total variation $\left(F_{7,57}=1.10, P>0.05\right)$. Station means for HSI ranged from $0.9 \%$ at Station 16 to $1.1 \%$ at Station 514 . Most individual values were $0.6-1.5 \%$, and only one fish from each of Stations 513 and 514 were $>1.5 \%$.

Splenosomatic index in carp differed significantly among stations $\left(F_{9,183}=6.26, P<0.01\right)$ and between genders $\left(F_{1,183}=11.81, P<0.01\right)$; ANOVA explained $31 \%$ of the total variation. In male carp, SSI station means ranged from $0.15 \%$ (Station 515 ) to $0.44 \%$ (Station 516). The lowest individual values $(0.01 \%)$ 
occurred at Stations 512 and 515; all others were $\geq 0.08 \%$ whereas individual male carp with SSI values $>0.5 \%$ were captured at Stations 64,514 , and 516. In female carp, SSI station means ranged from $0.13 \%$ at Station 512 to $0.28 \%$ at Station 514, and $90 \%$ of the individual values were between $0.08 \%$ and $0.32 \%$. Very low individual values $(0.01 \%)$ also characterized one female carp from each of Stations 512 and 515 whereas the maximum value $(0.80 \%)$ was represented by a fish from Station 64 . In contrast to carp, SSI differences in bass only approached statistical significance $\left(F_{8}, 63=1.92, P=0.07\right)$; ANOVA explained only $19 \%$ of the total variation. Values ranged from $0.01 \%$ in several fish from Stations 16,512 , and 513 to $0.8 \%$ in a largemouth bass from Station 16, but SSI varied greatly within stations. Station means ranged from $0.07 \%$ at Station 512 to $0.22 \%$ at Station 514 .

\subsubsection{Macrophage aggregates}

All three MA parameters differed significantly $(P<0.05)$ among stations in carp, but only MA-\# and MA- $\%$ differences were significant in bass (Table 5). In addition, age significantly $(P<0.05)$ affected MA-A and MA- $\%$ in both taxa (Table 5). With the genders combined, a 1-y increase in age was associated with a multiplicative change of 1.17 $(16 \%)$ in median MA-A in bass and 1.45 (44\%) in carp. For MA-\%, a 1-y increase in age was associated with a multiplicative change of $1.25(24 \%)$ in bass and $1.23(23 \%)$ in carp. Overall, age-adjusted MA parameters in carp were universally smallest at

Table 5

Age-adjusted station means for splenic macrophage aggregate (MA) parameters in carp and bass (Micropterus spp.; none collected at Stations $64,65,511,515$, or 516 )

\begin{tabular}{|c|c|c|c|c|c|c|}
\hline \multirow{2}{*}{$\begin{array}{l}\text { Sub-basin } \\
\text { and station }\end{array}$} & \multicolumn{3}{|l|}{ Carp } & \multicolumn{3}{|l|}{ Bass } \\
\hline & MA-\# (no. $\left./ \mathrm{mm}^{2}\right)$ & MA-A $\left(\mu \mathrm{m}^{2}\right)$ & MA- $\%(\%)$ & MA-\# (no. $\left./ \mathrm{mm}^{2}\right)$ & MA-A $\left(\mu \mathrm{m}^{2}\right)$ & MA- $\%(\%)$ \\
\hline \multicolumn{7}{|c|}{ Upper Rio Grande } \\
\hline 64 & $9.15 \mathrm{~cd}$ & $3431 \mathrm{bc}$ & $2.99 \mathrm{c}$ & - & - & - \\
\hline 63 & $8.97 \mathrm{bcd}$ & $4063 \mathrm{bcd}$ & $3.22 \mathrm{c}$ & $5.37 \mathrm{~b}$ & 3364 a & $1.66 \mathrm{abc}$ \\
\hline 516 & $6.54 \mathrm{bc}$ & 3989 bc & $2.46 \mathrm{bc}$ & - & - & - \\
\hline \multicolumn{7}{|c|}{ Middle Rio Grande/Pecos } \\
\hline 65 & 10.09 & $1911 \mathrm{ab}$ & $1.73 \mathrm{bc}$ & - & - & - \\
\hline 515 & $12.29 \mathrm{cde}$ & $3945 \mathrm{~cd}$ & $4.73 \mathrm{~cd}$ & - & - & - \\
\hline 514 & $3.46 \mathrm{a}$ & $1430 \mathrm{a}$ & $0.30 \mathrm{a}$ & $4.31 \mathrm{~b}$ & 2477 a & $0.89 \mathrm{bc}$ \\
\hline \multicolumn{7}{|c|}{ Lower Rio Grande } \\
\hline 513 & $2.95 \mathrm{a}$ & $1484 \mathrm{a}$ & $0.30 \mathrm{a}$ & $4.91 \mathrm{~b}$ & 2984 a & $1.25 \mathrm{bc}$ \\
\hline 16 & $8.35 \mathrm{bcd}$ & $1756 \mathrm{ab}$ & $1.11 \mathrm{bc}$ & $7.70 \mathrm{a}$ & 3685 a & $2.67 \mathrm{a}$ \\
\hline 511 & $7.82 \mathrm{bcd}$ & $3258 \mathrm{bc}$ & $1.79 \mathrm{bc}$ & - & - & - \\
\hline 512 & $14.51 \mathrm{e}$ & $6393 \mathrm{~d}$ & $8.49 \mathrm{~d}$ & $4.97 \mathrm{~b}$ & 3146 a & $1.44 \mathrm{bc}$ \\
\hline \multicolumn{7}{|l|}{$A N C O V A$} \\
\hline Model & $9.95^{* * a}$ & $9.18 * * \mathrm{a}$ & $14.50 * * \mathrm{a}$ & $5.73 * * \mathrm{~b}$ & $4.03 * * \mathrm{~b}$ & $5.23^{* * \mathrm{~b}}$ \\
\hline Station & $10.38 * * \mathrm{c}$ & $7.70 * *^{\mathrm{c}}$ & $13.19 * * \mathrm{c}$ & $5.65^{* *}{ }^{\mathrm{d}}$ & $1.10 \mathrm{~ns}^{\mathrm{d}}$ & $3.16^{* \mathrm{~d}}$ \\
\hline Age & $2.18 \mathrm{~ns}^{\mathrm{e}}$ & $15.41 * * \mathrm{e}$ & $18.01 * * \mathrm{e}$ & $1.68 \mathrm{~ns}^{\mathrm{f}}$ & $9.99 * * \mathrm{f}$ & $6.64^{* \mathrm{f}}$ \\
\hline
\end{tabular}

Shown are arithmetic mean MA density (MA-\#) and geometric mean MA area (MA-A) and percent tissue occupied (MA-\%) adjusted to the basin-wide mean age for each species (3.2 y for carp, 1.8 y for bass) using analysis-of-covariance (ANCOVA). Also shown are ANCOVA $F$ values and degrees-of-freedom $(d f)$ for the analyses $(* * P \leq 0.01 ; * 0.01<P \leq 0.05$; ns, $P>0.05)$. Within each column, means followed by the same letter are not significantly different ( $P>0.05$, Fisher's protected LSD).

${ }^{\mathrm{a}} d f=10,166$.

b $d f=5,68$.

c $d f=9,166$.

d $d f=4,68$.

e $d f=1,166$.

f $d f=1,68$. 
Stations 65, 513, and 514, and were largest at Station 512; all other stations were intermediate (Table 5). In addition, there was a gradient towards increasing values of all three MA parameters in carp with distance downstream in the lower RGB (Table 5). In bass, MA-\# and MA- $\%$ were significantly greater at Station 16 than at the other four sites from which bass were collected, but the latter did not differ (Table 5). As a group, differences among stations were less evident for bass than for carp (Table 5), but values of all MA parameters in bass were also relatively low at Station 514 and high at Station 16. Mean MA values (all three parameters) in bass from Station 512 were intermediate (Table 5), but the greatest individual MA-A and MA- $\%$ values for bass also occurred at Station 512 (data not shown).

\subsection{Reproductive biomarkers}

\subsubsection{Gonadosomatic index (GSI)}

ANOVA models containing the factors station, gender, stage, and their interactions were statistically significant for GSI in both carp $\left(F_{28,175}=16.26\right.$, $P<0.01)$ and bass $\left(F_{15,56}=8.73, P<0.01\right)$. In carp, the model explained $72 \%$ of the total variation; differences among stations were significant $\left(F_{6}, 175=4.58\right.$, $P<0.01)$, but not those between genders $\left(F_{1}, 163=\right.$ $0.19, P>0.05$ ). GSI in female carp ranged from $0.3 \%$ in a stage- 0 fish from Station 511 to $36.4 \%$ in a stage-2 fish from Station 16 (Fig. 6). Female carp from Stations 16,513 , and 514 had proportionately larger ovaries than those from most other stations, but there was wide variation that only partly corresponded to stage differences among the stations (Fig. 6). Carp ovaries from Station 511 were proportionally smaller than at most stations (Fig. 6), reflecting the earlier collection date at this site (Table 1). The distribution of GSI in male carp generally paralleled that of female carp and, like the females, was partly related to stage and collection date differences. Individual values ranged from $2.2 \%$ to $20.3 \%$ and were generally lowest at Station 511 (Fig. 6).

ANOVA accounted for $70 \%$ of total GSI variation in bass. Differences between genders were significant $\left(F_{1,56}=7.85, P<0.01\right)$, but among-station differences only approached significance $\left(F_{4,56}=2.42, P=0.06\right)$. All GSI values in bass were between $0.25 \%$ and $1.2 \%$ except for three fish-one (of two) stage-3 small-

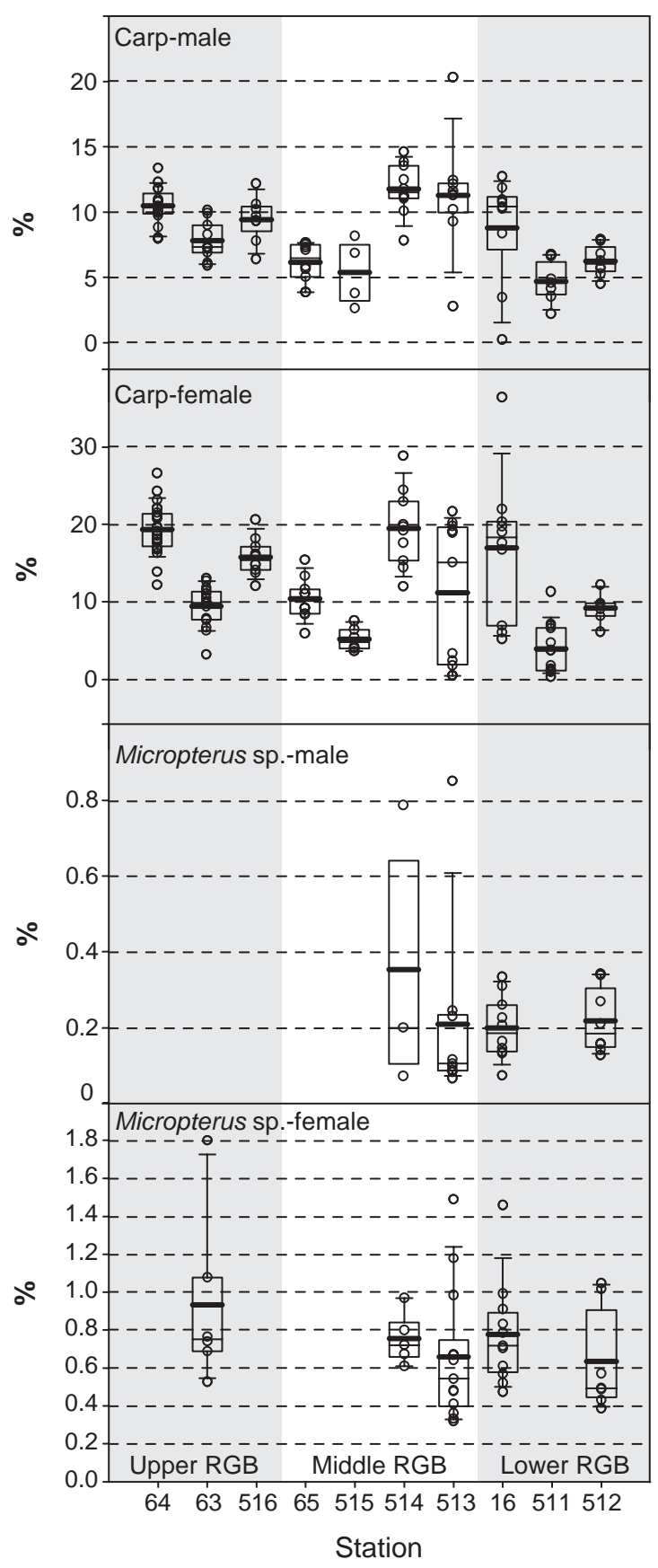

Fig. 6. Gonadosomatic index (GSI) values of male and female carp and bass (Micropterus sp.), by station. Shown for each group are points representing individual fish and the mean (heavy horizontal line), median (light horizontal line), interquartile range (box), and the 10th and 90th percentiles (whiskers). Stations are ordered from upstream to downstream and are grouped by sub-basin. 
mouth bass from Station $63(1.8 \%)$ and one stage-2 largemouth bass from each of Stations $16(1.5 \%)$ and $513(1.5 \%)$, and most values were between $0.35 \%$ and $1.0 \%$ (Fig. 6). The proportional size of the ovaries of the two stage-4 females from Station 514 was within the range spanned by other female bass (Fig. 6).

\subsubsection{Gonadal histopathology}

Ovary samples representing 113 female carp from all 10 stations were examined; only gonadal stages 0 2 were present. Stage 2 was predominant $(82 \%$ overall) and represented $64-100 \%$ of the female carp examined at most sites. Overall, female carp from Stations 511, 512, and 513 were less advanced (mostly stages 0 and 1) than at the other sites (mostly stages 1 and 2), reflecting the earlier collection date and lower GSI values at Station 511 (Table 1, Fig. 6). One 3-y old female carp from Station 64 could not be staged because no normal gonad tissue was observed; all ovarian follicles showed signs of degeneration, inflammation, and fibrosis.

Ovary samples representing 42 female bass from five stations were examined; all were largemouth bass except for two (stage-3) smallmouth bass from Station 63. Gonadal stages $0-4$ were represented; most (76\%) were in stage 1. Stage-0 (immature) female bass were collected exclusively at Station 512 (two of seven) whereas stage- 4 females (two of five) were obtained only at Station 514.

Varying degrees of oocyte atresia were detected in female carp and bass. ANOVA models that included terms for station, age, gonadal stage, and the interactions of these terms were statistically significant in both carp $\left(F_{25,71}=2.69, P<0.01\right)$ and bass $\left(F_{17,23}=25.41, P<0.01\right)$. ANOVA accounted for $49 \%$ of the total variation in carp and $95 \%$ in bass, but differences among stations were not significant in either taxon $(F<1.0, P>0.05)$ after accounting for all other factors. Atresia was typically $<20 \%$ in both taxa (Fig. 7); however, it was $50-80 \%$ in the two stage- 4 female bass from Station 514. Further histopathological examination revealed heavy microsporidian parasite infections within the oocytes of these two fish (Fig. 8A, B). Although the parasites did not appear to have penetrated the previtellogenic oocytes, the more advance oocytes were affected; they consequently appeared to have progressed to stage 4 but were not released. The oocytes observed were in various stages

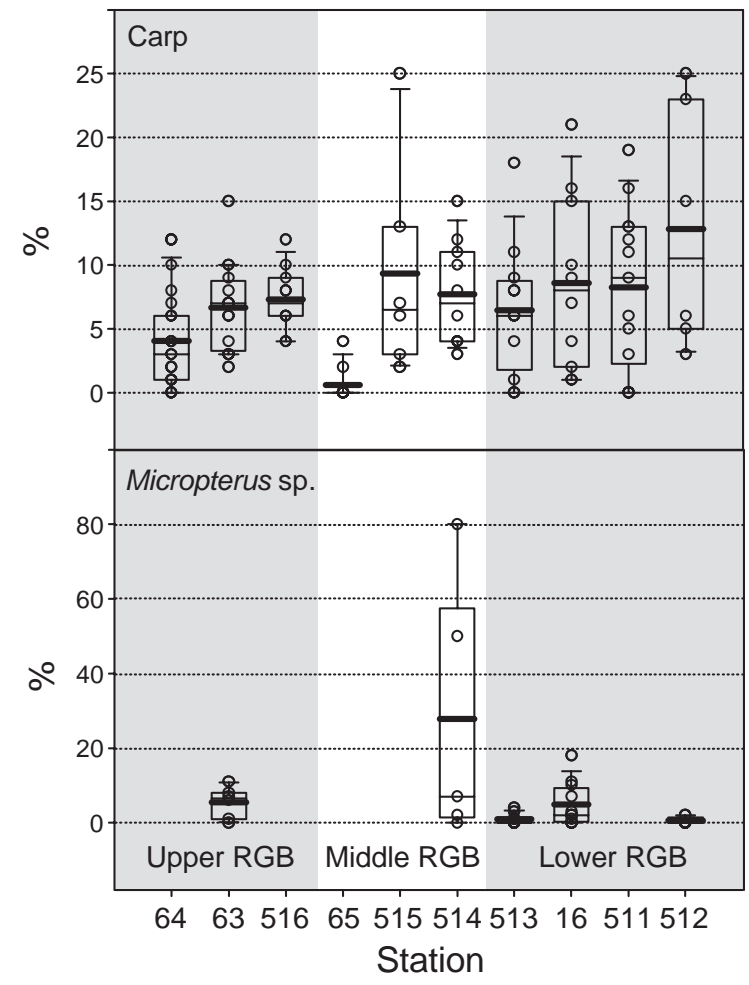

Fig. 7. Percentage of atretic oocytes in female carp and bass (Micropterus sp.), by station. Shown for each group are points representing individual fish and the mean (heavy horizontal line), median (light horizontal line), interquartile range (box), and the 10th and 90th percentiles (whiskers). Stations are ordered from upstream to downstream and are grouped by sub-basin.

of degeneration and necrosis and had begun to be reabsorbed (Fig. 8A).

Testes samples representing 92 male carp from all 10 stations were examined; gonadal stages 0 through 3 were represented. Most were in more advanced gonadal stages than females from the same sites; $89 \%$ were in stage 3 . As noted for females, most male carp from Station 511 were in less advanced gonadal stages (predominantly stage 2) than at the other stations, reflecting the earlier collection date and lower GSI values (Table 1, Fig. 6).

Testes samples representing 30 male bass from four stations were examined. All were largemouth except for one smallmouth bass from Station 514. Gonadal stages $1-3$, the latter only in the smallmouth bass, were represented; however, no gonad samples were obtained from the Station 63 male bass. As was true for male carp, the male bass examined were generally 


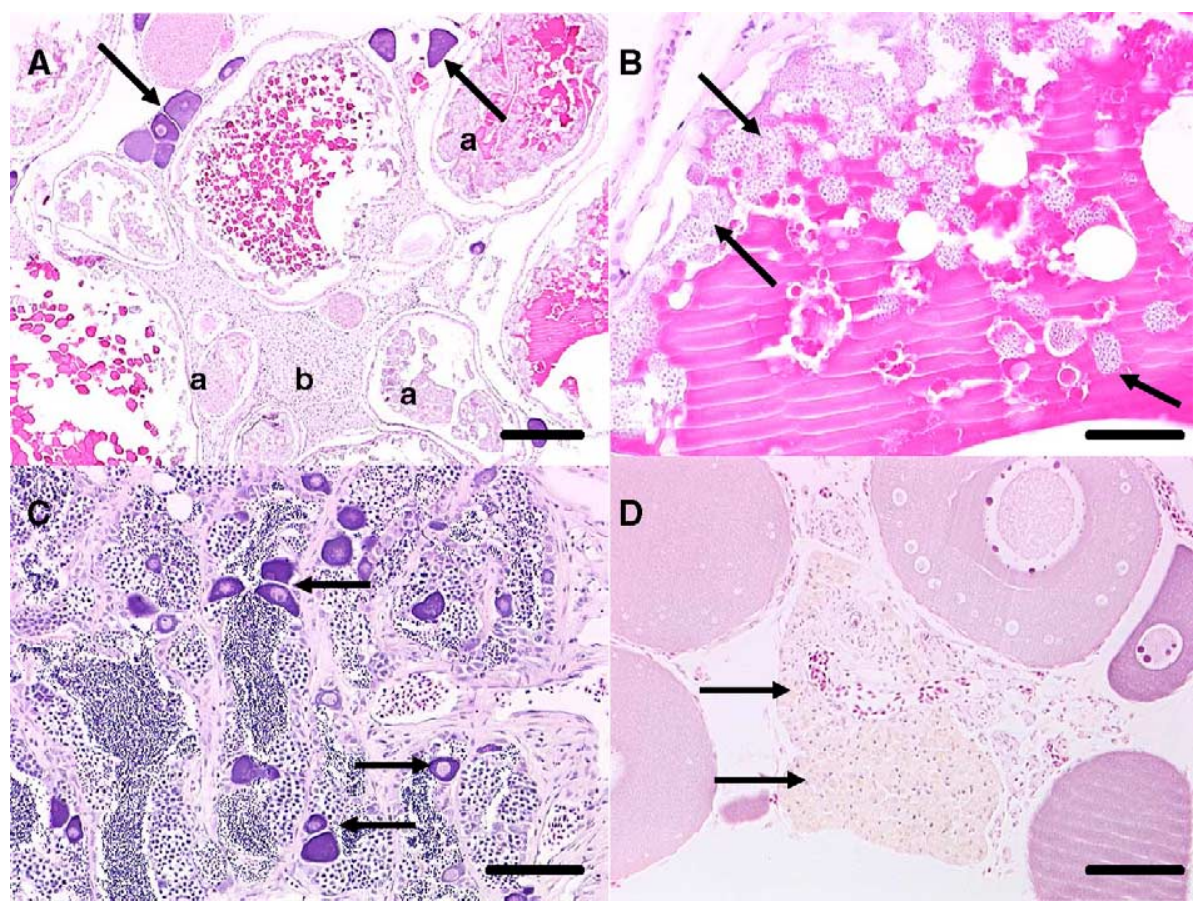

Fig. 8. A. Ovarian section of a largemouth bass from Station 514. Developing, vitellogenic oocytes in various stages of degeneration (a) and infected with an unidentified microsporidian parasite. Many ceroid/lipofuscin pigment accumulations (b) are also present. Previtellogenic oocytes (arrows) show no signs of infection. Scale bar $=200 \mu \mathrm{m}$. B. Higher magnification illustrating the presence of numerous microsporidian parasites (arrows). Scale bar $=50 \mu \mathrm{m}$. C. Intersex in male bass from station 512. Previtellogenic oocytes (arrows) can be observed within the testes. Scale bar $=100 \mu \mathrm{m}$. D. Pigmented cell accumulations (arrows) in the ovary of carp. The amount of these ceroid-lipofuscin pigments was rated on a scale of 0 (no pigment) to 4 (heavily pigmented). Scale bar $=100 \mu \mathrm{m}$. H and E stain.

in more advanced gonadal stages than females, and among-station differences were less evident. Ten male bass (all largemouth) representing three stations showed evidence of ovotestis as identified by the presence of developing oocytes in an otherwise normal male gonad (Fig. 8C). This intersex condition was detected in two of 10 fish from Station 16, four of eight from Station 512, and four of nine from Station 513. All of the intersex fish were identified as being in either gonadal stage 1 or 2 . None of three male bass from Station 514 (two largemouth, one smallmouth) examined were intersex. Ovotestis was not detected in male carp.

Pigmented cell accumulations were detected in the gonads of both male and female carp and bass from the RGB (Fig. 8D). The amount of these pigments varied from absent to moderately abundant, and tended to increase with age. Greater amounts were observed in females of both species, but differences among stations were not evident (data not shown).

\subsubsection{Vitellogenin (vtg)}

Concentrations of vtg in most male carp and bass were $<$ LOD $(0.005 \mathrm{mg} / \mathrm{mL}$ in carp, $<0.002 \mathrm{mg} / \mathrm{mL}$ in bass) and the data were not analyzed statistically. However, concentrations exceeded the LOD in at least one male carp from Stations 16, 63, 64, 511, 514, and 515 (data not shown). Except for one stage-2 fish from Station 511, all male carp in which vtg was detected were in gonadal stage 3 , and most of the measured concentrations were low $(<0.02 \mathrm{mg} / \mathrm{mL})$. Only three values exceeded $0.02 \mathrm{mg} / \mathrm{mL}$; these fish were from Stations $64(0.08 \mathrm{mg} / \mathrm{mL})$ and $511(0.18$ and $1.61 \mathrm{mg} /$ $\mathrm{mL}$ ). Vitellogenin was detected in only one of 27 male bass, a stage-2 fish from Station $513(3.15 \mathrm{mg} / \mathrm{mL})$.

In female carp and bass, ANOVA models that included the factors station, gonadal stage, age, and their interactions were highly significant; they explained $87 \%$ of the total variability in carp $\left(F_{25,69}=\right.$ $19.01, P<0.01)$ and $71 \%$ in bass $\left(F_{17,23}=3.50\right.$, $P<0.01)$, but differences among stations were not 
significant $(F<1.0, P>0.5)$ in either taxon after accounting for all other factors. In female carp, concentrations ranged from $<0.0005$ to $3.7 \mathrm{mg} / \mathrm{mL}$ and tended to increase with stage (Fig. 9). However, concentrations in stage-1 female carp from Station 513 were much lower $($ mean $=0.1 \mathrm{mg} / \mathrm{mL}$ ) than at all other sites (means $=1.2-2.0 \mathrm{mg} / \mathrm{mL}$ ). Concentrations of $\mathrm{vtg}$ in female bass ranged from $<0.005$ to $9.9 \mathrm{mg} / \mathrm{mL}$; the latter was a stage- 1 fish from Station 513 (Fig. 9). In addition to this fish, concentrations were also greater than most in a stage-2 bass from Station $513(7.2 \mathrm{mg} / \mathrm{mL})$. Conversely, the two stage-3 smallmouth bass from Station 63 contained $<0.005 \mathrm{mg} / \mathrm{mL}$ (Fig. 9). Concentrations were $2.9-3.3 \mathrm{mg} / \mathrm{mL}$ in the two parasite-infected largemouth bass from Station 514 (Fig. 8A, B).

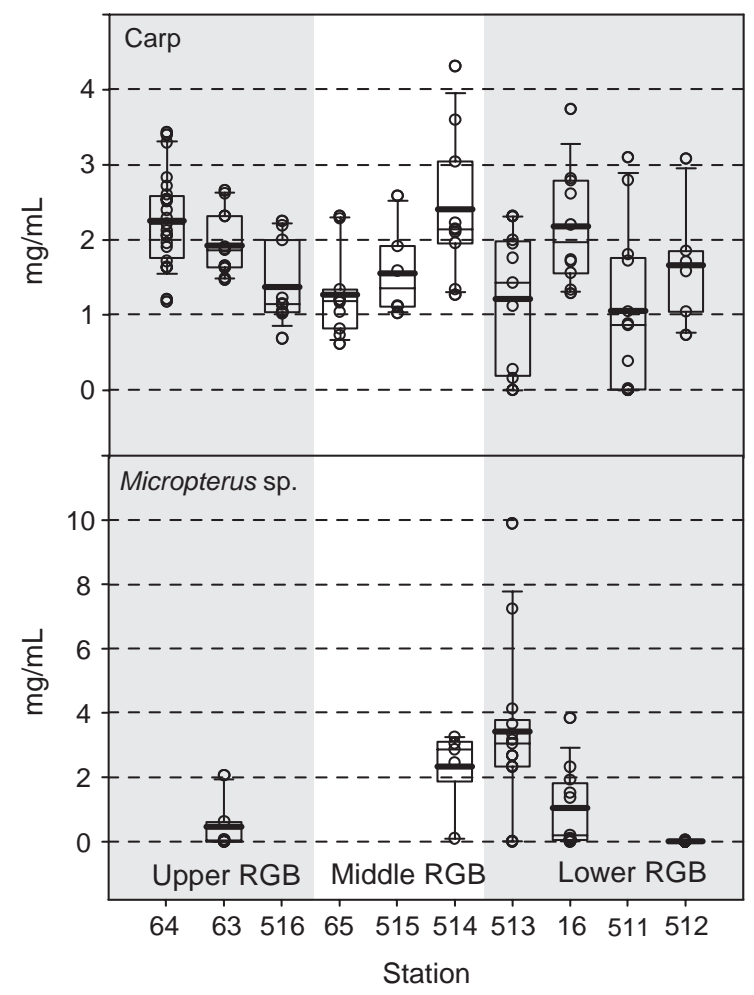

Fig. 9. Plasma vitellogenin (Vtg) concentrations in female carp and bass (Micropterus sp.), by station. Shown for each group are points representing individual fish and the mean (heavy horizontal line), median (light horizontal line), interquartile range (box), and the 10th and 90th percentiles (whiskers). Stations are ordered from upstream to downstream and are grouped by sub-basin.

\section{Discussion}

\subsection{Exposure indicators}

Of the elemental contaminants measured, only $\mathrm{Hg}$, As, and Se concentrations at some stations exceeded toxicity thresholds or were elevated relative to concentrations documented by previous investigations (Table 6). Elevated concentrations of all three had also been documented previously in the RGB. In contrast, concentrations of $\mathrm{Pb}, \mathrm{Cd}$, and $\mathrm{Zn}$ were either generally low or elevated in few samples.

It is generally accepted that most ( $>90 \%)$ of the $\mathrm{Hg}$ in fish occurs as the highly toxic methylmercury (Bloom, 1992; Southworth et al., 1995). Concentrations of total $\mathrm{Hg}$ in bass collected in 1997 from Station 63 were $0.30-0.50 \mu \mathrm{g} / \mathrm{g}$ (Fig. 2, Table 6), which is consistent with an existing consumption advisory for Elephant Butte Reservoir. Concentrations were lower, but nevertheless elevated relative to most sites, in other predatory fishes from Station 63. Elevated concentrations of $\mathrm{Hg}$ were also detected in bass from Stations 514 (Amistad Dam, TX) and 16 (Rio Grande at Mission, TX; Fig. 2, Table 6). In the past, elevated $\mathrm{Hg}$ concentrations were also reported in fish from the Rio Grande at El Paso (USEPA, 1992), but 1997 concentrations at Station 516 were universally $<0.06 \mu \mathrm{g} / \mathrm{g}$ (Fig. 2). Overall, Hg concentrations in bass from the RGB were generally similar to those reported for the Mississippi River Basin (MRB) in 1995 (Schmitt et al., 2002). Elevated concentrations of $\mathrm{Hg}$ are well documented in Elephant Butte Reservoir (Ong et al., 1991; Caldwell et al., 1999; Schmitt et al., 1999b; Canavan et al., 2000) and elsewhere in the RGB. Mercury, along with Se, is suspected of having caused the reproductive failure of peregrine falcons (Falco peregrinus) in Trans-Pecos Texas (Mora et al., 2002), where cinnabar was mined historically (Sharpe, 1980). Concentrations of $\mathrm{Hg}$ were $>0.3 \mu \mathrm{g} / \mathrm{g}$ in at least one sample from Stations 513 and 514, which may represent a threat to piscivorous birds, and at least one sample from all other stations except Stations 64 and 516 exceeded $0.1 \mu \mathrm{g} / \mathrm{g}$, a level that may represent a threat to piscivorous mammals (Yeardley et al., 1998; Wiener et al., 2002).

Fish are at greatest risk from environmental $\mathrm{Hg}$ during embryonic and larval stages, partially as a 
Table 6

Summary of chemical and biological findings indicative of exposure to contaminants, by sub-basin and station (designations are relative)

\begin{tabular}{llll}
\hline Sub-basin and station & Contaminants and EROD activity & Fish health indicators & Reproductive biomarkers \\
\hline $\begin{array}{l}\text { Upper Rio Grande } \\
64\end{array}$ & EROD (c) & (None observed) & $\begin{array}{l}\text { Vtg (mc), ovarian degeneration } \\
\text { (fc, } n=1)\end{array}$ \\
63 & Cr (mc), Hg (b, stb), EROD (c, b) & CF (c-), HAI (c, b) & $\begin{array}{l}\text { Vtg (fb-) } \\
\text { (None observed) }\end{array}$ \\
516 & DDE (c), EROD (c) & HAI (c) & \\
Middle Rio Grande & As (mwb, mc), Se (mwb, mc, fc), & CF (c-) & (None observed) \\
65 & EROD (c, b) & EL (c), HAI (c) & (None observed) \\
515 & Se (c) Stage (fb); atresia (fb); ceroid (fb); \\
514 & As (c), Se (c, b), Hg (b), EROD (c) & GSI (fb); ovarian parasites
\end{tabular}

Lower Rio Grande
513
16
511

512

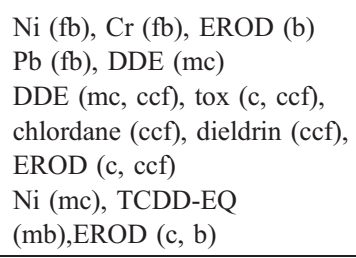

\author{
HAI (c, b) \\ (none observed) \\ HAI (c) \\ Vtg (mb), ovt (mb)
Ovt (mb)
Stage (c), vtg (mc) \\ CF (c-), HAI (b-), \\ MA (mc, fc), EL (b)
}

Male (m) and female (f) bass and carp were collected from all sites unless otherwise indicated. If gender is not specified, then the indicated condition was present in both. Additional abbreviations. DDE, $p, p^{\prime}$-DDE; tox, toxaphene; chlordane, sum of cis- and trans-chlordanes and nonachlors; oxychlordane; and heptachlor epoxide; TCDD-EQ, dioxin-like activity as determined by H4IIE bioassay; As, arsenic; Cr, chromium; $\mathrm{Hg}$, mercury; $\mathrm{Pb}$, lead; $\mathrm{Ni}$, nickel; EROD, hepatic ethoxyresorufin $O$-deethylase activity; EL, external lesions; $\mathrm{CF}$, condition factor; vtg, vitellogenin; HAI, health assessment index; SSI, splenosomatic index; GSI, gonadosomatic index; ovt, ovotestis; MA, macrophage aggregates (one or more parameters); b, bass (Micropterus sp.); c, carp (Cyprinus carpio); ccf, channel catfish (Ictalurus punctatus); wb, white bass (Morone chrysops); stb, striped bass (Morone saxatilis); - indicates that the response or condition was smaller or lower than most; all others larger or greater.

consequence of maternal transfer (Wiener and Spry, 1996). Behavioral effects in laboratory studies have been documented in fish containing whole-body $\mathrm{Hg}$ concentrations as low as $0.7 \mu \mathrm{g} / \mathrm{g}$ (Kania and O'Hara, 1974), but other studies have shown behavioral effects only at total $\mathrm{Hg}$ concentrations of 5-10 $\mu \mathrm{g} / \mathrm{g}$ (Wiener and Spry, 1996; Wiener et al., 2002). Juvenile grayling (Thymallus thymallus) containing wholebody concentrations of $0.27 \mu \mathrm{g} / \mathrm{g}$ resulting from dietary methylmercury exposure experienced permanent impairment of feeding efficiency and competitive ability (Fjeld et al., 1998). In female fathead minnows (Pimephales promelas), dietary methylmercury concentrations of $0.87 \mu \mathrm{g} / \mathrm{g} \mathrm{dw}$ (about $0.17 \mu \mathrm{g} / \mathrm{g}$ ww assuming $80 \%$ moisture) increased whole-body $\mathrm{Hg}$ concentrations by more than 10-fold, suppressed hormone levels, and inhibited gonadal development relative to controls (Drevnick and Sandheinrich,
2003). It therefore appears that the greatest concentrations observed in fish from the RGB $(\sim 0.5 \mu \mathrm{g} / \mathrm{g})$ may be sufficiently high to represent a risk to the fish themselves. However, as noted by Wiener et al. (2002), many factors can contribute uncertainty to the estimation of toxicity thresholds from tissue concentrations, and further study would be necessary to document such effects in RGB fish.

Comparatively high Se concentrations $(>1.0 \mu \mathrm{g} / \mathrm{g})$ characterized fish from Stations 65, 514, and 515 (Fig. 2, Table 6) and confirmed previous findings for the central RGB. The Se concentration in Morone sp. from Station 65 was lower in 1997 than in previous collections at this site, however (Schmitt et al., 1999b). Concentrations of Se in all RGB fish were considerably lower than the 3-5 $\mu \mathrm{g} / \mathrm{g}$ reported for a site in the MRB with a history of contamination from irrigated agriculture that was sampled in 1995 
(Schmitt et al., 2002, 2004). Numerous studies have demonstrated that the diet is the primary route of Se exposure and toxicity in aquatic vertebrates (see reviews by Lemly (2002) and Hamilton (2004)). Whole-body Se concentrations of $8-16 \mu \mathrm{g} / \mathrm{g} \mathrm{dw}$ (1.6-3.2 $\mu \mathrm{g} / \mathrm{g}$ ww assuming $80 \%$ moisture) have been associated with reproductive failure in fathead minnow (Schultz and Hermanutz, 1990) and bluegill (Lepomis macrochirus; Coyle et al., 1993; Gillespie and Baumann, 1986; Hermanutz et al., 1992); in fish, maternal transfer to eggs and embryos represents an important route of Se exposure. Conditions such as swelling of the gill lamellae, elevated lymphocyte counts, corneal cataracts, exophtalmus, pathological alterations of liver, kidney, heart and ovary, and teratogenic deformities of the spine, head, mouth, and fins have also been documented as a consequence of chronic Se exposure (Lemly, 2002; Hamilton, 2004). Because of its high toxicity and bioaccumulation potential in aquatic ecosystems, the thresholds for toxicity associated with concentrations in whole fish are comparatively low: $4 \mu \mathrm{g} / \mathrm{g} \mathrm{dw}(0.8 \mu \mathrm{g} / \mathrm{g}$ ww assuming $80 \%$ moisture) for larval fish toxicity (through maternal transfer) and $3 \mu \mathrm{g} / \mathrm{g} \mathrm{dw}(0.6 \mu \mathrm{g} / \mathrm{g}$ ww) for piscivorous wildlife (Lemly, 1996, 2002). Mean Se concentrations in fish from Stations 65, 514, and 515 and individual fish from several other sites exceeded these criteria.

The toxicity of Se may be mediated somewhat by dietary As (Hamilton et al., 2002). Concentrations of both As and Se are naturally elevated in some parts of the RGB and may be further increased by leaching due to irrigation, and both As and $\mathrm{Se}$ are released during the combustion of fossil fuels. Arsenic is also discharged from sewage treatment plants in areas of the RGB where groundwater concentrations are naturally elevated (Wilcox, 1997), and is released during the smelting of metals. Arsenic-containing pesticides and defoliants, which are used extensively in parts of the RGB, are also a significant source. Arsenic accumulates in planktivorous fishes and higher trophic level species that consume them (e.g. Hunter et al., 1981), and concentrations may be elevated in fish from impoundments (Schmitt et al., 2002; Schmitt, 2004). Consistent with previous studies, As concentrations were comparatively high $(0.37-0.55 \mu \mathrm{g} / \mathrm{g})$ in carp and other fishes from Stations 65 and 514 (Fig. 2; Table 6); comparable concentrations were reported in fish collected in 1995 from impoundments in the southwestern parts of the MRB (Schmitt et al., 2002; Schmitt, 2004). At least one sample from every site sampled in 1997 except Station 64 contained $>0.2 \mu \mathrm{g} / \mathrm{g}$ (Fig. 2); however, As in freshwater fish is generally not believed to represent a hazard to the fish or to fish-eating wildlife because the As occurs in a comparatively non-toxic form (Law, 1996).

Other than $\mathrm{Hg}$ and As, concentrations of elemental contaminants associated with mining in the RGB (e.g., $\mathrm{Pb}, \mathrm{Cd}$, and $\mathrm{Zn}$ ) were comparatively low. The exception was $\mathrm{Cd}$ in one carp sample from Station 64 (Rio Grande at Alamosa, CO; Fig. 2), which was greater than at most other RGB sites but nevertheless low compared to concentrations measured elsewhere (Schmitt, 2004). Station 64 is downstream from the Crede Mining District, where elevated concentrations of metals from historical mining and related activities have been reported previously in water, sediment, and biota (e.g., Carter and Anderholm, 1997; Levings et al., 1998).

Of the organochlorine compounds measured, only residues derived from the pesticides DDT (as $p, p^{\prime}$ DDE), chlordane, dieldrin, and toxaphene were detected. Residues were present at relatively high concentrations in fish from lower RGB sites, with greatest concentrations occurring in channel catfish from Station 511 (Arroyo Colorado at Harlingen, TX; Fig. 3, Table 6). Channel catfish from Station 511, along with blue catfish from Station 516 and carp from Stations 513 and 514, contained greater amounts of lipid $(9-11 \%)$ than all other samples analyzed (typically $2-5 \%$; data not shown), which may partly explain the comparatively high residue concentrations in some of these samples; however, many other factors including trophic position, growth rate, and reproductive status may also be involved.

Although the use of DDT was outlawed in the United States in 1972, it continued to be used in Mexico through 1997 (Environmental Health Perspectives (EHP), 1997; Mora, 1997). We detected DDT-derived residues at all sites; however, most of this material was $p, p^{\prime}$-DDE, indicating weathered material rather than the influx of new insecticide (Aguillar, 1984; Schmitt et al., 1999b). Except for Station 516 (Rio Grande at El Paso, TX), p, $p^{\prime}$-DDE concentrations in fish from sites in the upper and 
middle RGB were low, as they also were in 19861992 samples analyzed by others (Ong et al., 1991; Carter and Anderholm, 1997; Levings et al., 1998). In contrast, concentrations at Station 16 (Rio Grande at Mission, TX), on the lower Rio Grande, remained relatively high $(0.32-0.52 \mu \mathrm{g} / \mathrm{g})$ in 1997 (Fig. 3). Nevertheless, even these concentrations represent a substantial decrease since the 1980s, when they were consistently $>1.0 \mu \mathrm{g} / \mathrm{g}$ (Schmitt et al., 1999b). Some concentrations of $p, p^{\prime}$-DDE remained sufficiently elevated to represent a threat to fish-eating birds; at least one sample from Stations 16, 513, and 516 contained $>0.15 \mu \mathrm{g} / \mathrm{g}$, which is potentially toxic to the most sensitive avian species (Anderson et al., 1975), and all channel catfish samples from Station 511 (Arroyo Colorado at Harlingen, TX) contained 1-2 $\mu \mathrm{g} / \mathrm{g}$. The latter concentrations are potentially toxic to most avian wildlife (Blus, 1996). Wildlife criteria for total DDT as low as $0.20 \mu \mathrm{g} / \mathrm{g}$ ww have been proposed (Newell et al., 1987), and concentrations of $0.4-0.5 \mu \mathrm{g} / \mathrm{g}$ have been associated with reproductive toxicity in several species of fish (see review by Jarvinen and Ankley, 1999).

The greatest $p, p^{\prime}$-DDE concentrations in fish from the lower RGB $(\sim 1-2 \mu \mathrm{g} / \mathrm{g})$ were similar to those in fish collected in 1995 from the largest rivers draining cotton-farming regions of the lower Mississippi River valley, but greater concentrations $(5-8 \mu \mathrm{g} / \mathrm{g})$ were detected in smaller rivers and streams (Schmitt et al., 2002 , 2004). In contrast, the proportional concentrations of $o, p^{\prime}$-DDT homologs were not great enough to indicate industrial inputs from manufacture or formulation at any RGB sites; traces of $o, p^{\prime}$-DDD were detected exclusively in the channel catfish from Station 511. Residues of $o, p^{\prime}$-DDT homologs were historically considered to be comparatively benign; however, recent studies have shown that $o, p^{\prime}$-DDD is weakly estrogenic (Ackerman et al., 2002; Guillette et al., 1996; Toppari et al., 1996), as are many other pesticides and their metabolites (e.g., Tyler et al., 1998). The total risk of the DDT-derived residues present in fish from the lower RGB is therefore unknown. In general, however, 1997 findings for DDT in the lower RGB and at El Paso confirmed the results of other recent investigations of this area (USEPA, 1992; TNRCC, 1994a,b, 1997; Davis et al., 1995; Moring, 1999; Wainwright et al., 2001), and the Arroyo Colorado and nearby waters remain under a fish consumption advisory due to contamination by $p, p^{\prime}$-DDE and other pesticides. In addition, it also important to note that although high by contemporary standards, even the greatest $1997 p, p^{\prime}$-DDE concentrations in the lower RGB represent a substantial decline relative to the $1980 \mathrm{~s}$, when fish from some sites contained $>20 \mu \mathrm{g} / \mathrm{g}$ (White et al., 1983; Gamble et al., 1988).

Chlordanes were among the few other organochlorine chemical residues detected at potentially toxic concentrations in 1997. During the 1970s, traces of chlordane components $(\leq 0.01-0.3 \mu \mathrm{g} / \mathrm{g})$ were detected in some samples from Stations 63, 64, and 65 (Schmitt et al., 1999b) but a trend toward nondetections that began in the 1980s continued at these stations; 1997 concentrations of all six components measured were $<0.01 \mu \mathrm{g} / \mathrm{g}$ in all samples. Traces of chlordane constituents were detected in fish samples from some sites in the upper RGB sampled in 19861992 (Ong et al., 1991; Carter and Anderholm, 1997; Levings et al., 1998). At Station 16, concentrations of individual chlordane components ranged from $<0.005$ to $0.09 \mu \mathrm{g} / \mathrm{g}$ in $1976-1986 \mathrm{NCBP}$ collections (Schmitt et al., 1999b), but were slightly lower in 1997; all component concentrations were $\leq 0.01 \mu \mathrm{g} / \mathrm{g}$ except for two trans-nonachlor values. In contrast, 1997 chlordane concentrations were $>0.1 \mu \mathrm{g} / \mathrm{g}$ in channel catfish from Station 511 (Fig. 3, Table 6), where they were also elevated in all recent and contemporaneous studies (USEPA, 1992; TNRCC, 1994a,b, 1997; Davis et al., 1995). Chlordane is part of the fish consumption advisory for the Arroyo Colorado and nearby waters; 1997 concentrations also remained sufficiently elevated to also represent a threat to fisheating wildlife. In addition, the proportional concentrations of cis-chlordane remained high relative to those of trans-nonachlor and other components at Station 511, which may have resulted from more recent inputs; U.S. use of chlordane was curtailed in the 1970s but it continued to be used in Mexico through 1997 (EHP, 1997; Mora, 1997). Elsewhere in the U.S., residual chlordane from historical use has been implicated in comparatively recent wildlife kills (Stansley and Roscoe, 1999), which illustrates the continuing risk represented by this obsolete pesticide.

Toxaphene concentrations were historically elevated in the lower RGB, but those in the upper basin were low; concentrations did not exceed $0.3 \mu \mathrm{g} / \mathrm{g}$ at 
Stations 63, 64, and 65 during the 1970s, and levels in all but one sample collected in the 1980 s were $\leq 0.1$ $\mu \mathrm{g} / \mathrm{g}$. In 1997 toxaphene was not detected at these sites (Fig. 3). Residues were also not detected in fish collected from the upper RGB in 1986-1992 (Ong et al., 1991; Carter and Anderholm, 1997; Levings et al., 1998) or from the transboundary segments of the RG during the mid-1990s (TNRCC, 1994a,b, 1997). In contrast, toxaphene concentrations at Station 16 were elevated in the $1970 \mathrm{~s}(0.3-1.4 \mathrm{ig} / \mathrm{g})$ but decreased through the $1980 \mathrm{~s}$ (to $0.2-0.3 \mu \mathrm{g} / \mathrm{g}$ in 1986). Wainwright et al. (2001) also reported concentrations as great as $0.3 \mu \mathrm{g} / \mathrm{g}$ in carp and $4.4 \mu \mathrm{g} / \mathrm{g}$ in green-backed heron (Butorides virescens) eggs from resacas (oxbow lakes) and settling basins in the lower RGB. All 1997 samples from Station 16 contained $\leq 0.11 \mu \mathrm{g} /$ $\mathrm{g}$; however, toxaphene concentrations were $0.5-2.5$ $\mu \mathrm{g} / \mathrm{g}$ in fish from Station 511 (Fig. 3), which is consistent with recently reported concentrations at this site (TNRCC, 1994b, 1997; Davis et al., 1995). These values are also similar to those in fish collected from cotton-farming regions of the MRB in 1995 (Schmitt, 2002a; Schmitt et al., 2002) and may be sufficiently high to represent a threat to fish; however, the toxicity of weathered toxaphene is highly variable and cannot be determined from the 1997 data, which is based on a relatively low-resolution analytical procedure (Bidleman et al., 1993; Muir et al., 1999). Toxaphene is also a component of the fish consumption advisory for the Arroyo Colorado and nearby waters.

In 1997, PCBs were not detected $(<0.05 \mu \mathrm{g} / \mathrm{g})$ in any samples from the RGB, and the greatest TCDDEQ concentrations were only 3-6 pg/g (in fish from Stations 511 and 512). Concentrations of TCDD-EQ at reference sites in previous studies ranged from below detection to $6 \mathrm{pg} / \mathrm{g}$ (Giesy et al., 1995; van den Heuvel et al., 1996; Schmitt et al., 2002; Whyte et al., 2004), and $6 \mathrm{pg} / \mathrm{g}$ has been suggested as a threshold for toxicity to fish-eating wildlife (Giesy et al., 1995). This threshold was attained by one bass sample from Station 512 (Table 6). Our results were consistent with recent and historic findings of generally low levels of PCB and dioxin contamination in the RGB (Ong et al., 1991; USEPA, 1992; TNRCC, 1994a,b, 1997; Carter and Anderholm, 1997; Levings et al., 1998; Schmitt et al., 1999b; Wainwright et al., 2001). The toxicity of weathered PCBs varies greatly depending on the relative abundance of the congeners present (van den Berg et al., 1998), which cannot be determined from the low-resolution methods used to analyze the RGB samples. Nevertheless, the universally low total PCB and TCDD-EQ concentrations indicate a comparatively low level of risk to wildlife from TCDD and similar compounds at the RGB sites investigated.

Based on data from the MRB and the extensive review of the literature conducted by Whyte et al. (2000), Schmitt et al. (2002) considered the normal ranges of hepatic EROD expression to be $0-4 \mathrm{pmol} /$ $\mathrm{min} / \mathrm{mg}$ in female carp, $0-6 \mathrm{pmol} / \mathrm{min} / \mathrm{mg}$ in male carp, $0-16 \mathrm{pmol} / \mathrm{min} / \mathrm{mg}$ in female bass and 0-22 $\mathrm{pmol} / \mathrm{min} / \mathrm{mg}$ in male bass. Relative to these ranges, EROD rates in at least one fish were induced above basal levels and therefore indicative of exposure to exogenous AhR agonists at all RGB stations except Station 515, and were greatest on average at Stations 511 and 512 in all species (Tables 4, 6). Geometric station means for carp exceeded the indicated criteria in one or both genders at Stations 511,512 , and 516, and at least one carp exceeded the criteria at all sites except Stations 16 and 515 (Table 4). In bass, EROD activity in most individual fish and all geometric means exceeded the previously cited criteria at all sites from which they were collected (Table 4). Although basal rates have not been determined for channel catfish, EROD rates were also greater at Station 511 than at Station 516 and exceed previously reported rates for this species (Whyte et al., 2000; Schmitt et al., 2002). Given the low concentrations of total PCBs $(<0.05 \mu \mathrm{g} / \mathrm{g})$ and TCDD-EQ $(\leq 6 \mathrm{pg} / \mathrm{g})$ at all sites, the EROD findings indicate that fish from most RGB stations had been exposed to polycyclic aromatic hydrocarbons (PAH). In addition, there was a general gradient of increasing EROD activity from upstream to downstream. Moring (1999) detected PAH in SPMD samples from five of six transboundary stations sampled in July-August 1997; both the concentrations and numbers of compounds detected also generally increased from upstream to downstream. Although PAH emanate from a variety of other sources (Schmitt, 1998), the extensive oil and gas extraction and transportation industry of the RGB cannot be overlooked as a potential source of these compounds and the cause of the EROD induction we detected. 


\subsection{Fish health indicators}

Of the quantitative fish health biomarkers we employed, CF, HSI, and HAI have been the most widely used and discussed in the literature. Condition factors of 1.0-2.0 for carp and bass, the range of most RGB values, are typical for these taxa (Carlander, 1969, 1977; Blazer et al., 2002). The HSI range for RGB bass was $0.6-1.5 \%$, which is also typical for Micropterus sp. Liver enlargement has been reported in largemouth bass from PCB-contaminated sites (Adams and McLean, 1985) as well as in other fishes exposed to contaminants in both field and laboratory studies (see review by Dethloff and Schmitt, 2000 and subsequent studies by Sepúlveda et al., (2001, 2003). The liver represented $0.5-1.5 \%$ of body weight in most male and female bass collected from the MRB in 1995, but enlarged livers were detected in bass from a few PCB- and pesticide-contaminated sites (Blazer et al., 2002). In contrast, HSI values in bass were not indicative of liver enlargement at any RGB site (Table 6). According to Gingerich (1982), the liver constitutes $1-2 \%$ of body weight in most fishes. Most RGB bass had proportionally smaller livers, as did most of those collected from the MRB in 1995 (Blazer et al., 2002).

In the RGB, mean CF and SSI in carp were greatest at Stations 513 and 514, and individual carp with relatively high CFs were captured at Stations 16, 64, 511, and 514 (Table 6). Spleen size was also relatively large in individual carp from Stations 16, 64, 514, and 516. In bass, mean SSI was also relatively high at Station 514, and individual fish with relatively large spleens were captured from Stations 16, 513, and 514. Spleen enlargement is often associated with infections. In contrast, both carp and bass from Station 512 had relatively small spleens, a condition that has been associated with contaminant exposure (Blazer et al., 2002).

Background information on external lesions, MA parameters, and SSI are either not completely relevant because most studies conducted to date investigated only marine or estuarine fish or because information is not available. For external abnormalities, difficulties in comparing results among studies arise from probable systematic error caused by increasing familiarity of field personnel as the study progresses (Leonard and Orth, 1986) and from differences in the anomalies characterized and recorded (Karr, 1981; Fournie et al., 1996; Sanders et al., 1999). We used criteria modified only slightly from the 1995 MRB investigation (Blazer et al., 2002), so the results of these surveys are comparable. Our external lesion procedure is also similar to the deformities, erosion, lesions, and tumors (DELT) component of the Index of Biotic Integrity (IBI; Karr, 1981; Sanders et al., 1999), which is widely employed. In general, we noted sites at which $\geq 50 \%$ of carp, bass, or all fish had grossly visible lesions; in the RGB these included Station 515 for carp and all fish, and Stations 512 and 514 for bass (Table 6). Overall, lesion frequencies were similar in the RGB and MRB (Blazer et al., 2002).

The HAI had not been used with carp prior to the 1995 study (Blazer et al., 2002); however, it was used to assess largemouth bass populations in Tennessee Valley Authority reservoirs (Adams et al., 1993) and in the Catawba River system of North and South Carolina (Coughlan et al., 1996). In the latter studies, a positive linear relation between average fish weight or age and HAI score was noted, and Coughlan et al. (1996) suggested that only bass of 250-450 mm (TL) be included in comparisons. Approximately $90 \%$ of the bass collected in the RGB met these criteria. Based on previous findings (Adams et al., 1993; Coughlan et al., 1996) and conservative precedent (Blazer et al., 2002), we assumed that mean HAI values $\leq 20$ indicated unimpacted or minimally impacted sites, values $>50$ indicated intermediate sites, and values $>70$ indicated heavily impacted sites. Carp and bass from many MRB sites sampled in 1995 averaged $>70$, and most station means were $>50$ (Blazer et al., 2002). In the RGB, HAI scores were similar; the mean for carp from Station 515 was 70, and those for Stations 511, 513 , and 516 were $>50$ (Fig. 4, Table 6). The mean for bass from Station 63 was also 70, and those from Stations 16, 513, and 514 were $>50$. Collectively, these findings and the frequencies of external lesions indicate some degree of degradation at many sites in both basins.

A recent study conducted in the Gulf of Mexico established a value of $>40$ splenic $\mathrm{MA} / \mathrm{mm}^{2}$ in at least one fish from a site as a threshold indicative of possible effects due to hypoxia or sediment contamination (Fournie et al., 2001). Blazer et al. (2002) also 
used this value as a benchmark for carp and bass in the MRB; however, it was derived for marine and estuarine fishes and it is important to note that additional research on freshwater species, particularly bass and carp, is necessary to determine a threshold for possible effects. It is also important to note that using only MA-\# does not take into consideration that there can be a few very large aggregates. Regardless, no fish from the RGB contained $>40$ splenic MA $/ \mathrm{mm}^{2}$ (MA-\#), all carp except one from Station 512 contained $<20 \mathrm{MA} / \mathrm{mm}^{2}$, and all bass had $<12 \mathrm{MA} /$ $\mathrm{mm}^{2}$. These relatively low values were generally about the same as what was reported for carp and bass collected from the MRB in 1995 (Blazer et al., 2002). Although low relative to the $40 \mathrm{MA} / \mathrm{mm}^{2}$ criterion of Fournie et al. (1996, 2001), values of MA-\# and the other MA parameters tended to be lowest at Stations 513 and 514 and greatest at Station 512. In addition, the MA- $\%$ values for Station 512 carp exceeded those for any other station sampled in either the RGB or the MRB (Blazer et al., 2002). Increased numbers of MAs have been associated with exposure of fish to contaminants including crude oil and As [see review by (Blazer and Dethloff, 2000)], both of which occur in the RGB. Macrophage aggregate numbers, area, and volume may also increase as a consequence of bacterial infection (Matsche and Grizzle, 1999). In contrast to our findings, Mora et al. (2001) reported that fish of several species obtained from lower RGB resacas contained high numbers of MAs. However, it is important to note that their sample numbers were small and their fish were not aged, which makes direct comparisons with the 1997 data problematic (Blazer et al., 1987).

As noted for sites in the MRB sampled in 1995 (Blazer et al., 2002), we did not observe a high incidence of confirmed tumors or other grossly visible indications that fish were exposed to elevated concentrations of toxic chemicals at any RGB sites (e.g., Baumann et al., 1991). Papillomas (benign tumors of the skin) were noted in two fish. Although papillomas have been associated with viral infections in carp (Hedrick et al., 1990), a higher prevalence in other fish species has been reported in populations exposed to industrial or sewage effluents (Kortet et al., 2002). We also noted an apparent proliferation of ectopic thyroid follicles within the posterior kidney that was not evident during gross examination in carp from sites in the lower and middle RGB. Similar observations were made for carp from some sites in the lower MRB sampled in 1995 (Blazer et al., 2002). Thyroid hyperplasia in fish and other organisms has been induced by exposure to a wide array of contaminants (e.g., Patiño et al., 2003 and studies cited by Blazer et al., 2002) and, along with endpoints indicative of thyroid function and homeostasis (see review by Brown et al., 2004), may represent a potential biomarker for contaminant effects on thyroid function. However, the effects of confounding factors such as area of kidney sampled and fish age on thyroid histopathology need to be evaluated.

Lipopigment (ceroid/lipofuscin) deposition in liver, gonad, and other tissues was observed in fish from many sites. These pigments, which represent peroxidized forms of lipid, tend to accumulate with age (Hammer and Braum, 1988) and may represent oxidative damage resulting from contaminant exposure. A variety of contaminants, including PAH (Au et al., 2004), organochlorine insecticides (Nowak and Kingsford, 2003), and PCBs (Kohler, 1990), have been shown to increase the amounts of lipopigments in various fish tissues. So also have bacterial infection (Matsche and Grizzle, 1999). Many environmental and physiological variables can also influence pigment accumulation (Hill and Womersley, 1993). Pigment accumulations in the gonads of carp and bass at many RGB sites tended to increase with fish age. The quantities observed were generally low, indicating a low level of oxidative stress. In contrast, fish of several species obtained from lower RGB resacas and settling basins by Mora et al. (2001) in 1996 contained high concentrations of ceroid/lipofuscin pigments. However, and as noted with regard to MAs, these fish were not aged and it is consequently difficult to compare the studies.

\subsection{Reproductive biomarkers}

Reproductive biomarker measurements of note were determined primarily through comparison with the 1995 MRB study (McDonald et al., 2002) and the literature; McDonald et al. (2002) documented the importance of controlling for stage differences in such comparisons. Both male and female carp from Station 
511 (AC at Harlingen, TX) were in earlier gonadal stages and had lower GSIs than those from all other stations, probably because they were collected several weeks before all other stations in the lower RGB. Bass from Station 512 were also in slightly earlier stages than those from the other three stations from which bass gonads were obtained for examination (only females from Station 63). Fish were collected in the RGB over a 4-month period; although most were obtained between late September and early December 1997, Station 65 was not sampled until late January 1998. Nevertheless, carp from Station 65 were in the same stages (females all stage 2, males all stage 3 ) as nearby stations that were sampled earlier. Thus, it does not appear that reproductive biomarker results at sites other than Station 511 were confounded by sampling period differences.

For atresia, McDonald et al. (2002) defined high percentages as $\geq 25 \%$ for female carp and $>10 \%$ for bass. In the RGB, atresia was $<20 \%$ in most female carp and $<10 \%$ in most female bass. High individual values occurred in both carp and bass from Station 16, but only in bass from Stations 512 and 514 (Table 6). Values in female bass from Station 514 were greater than any reported in bass previously but were related to a parasite infection; and the ovaries of one female carp from Station 64 contained no normal tissue, only necrotic, degenerated follicles. Elevated rates of atresia in fish have been associated with exposure to Se (Sorensen, 1988) and other contaminants (e.g., Cross and Hose, 1988), but may also be caused by environmental factors other than chemical exposure (e.g., June, 1970, 1977).

To our knowledge, the background occurrence of intersex male bass has not been established. We therefore recorded where they were found and at what frequency, but have no criteria for comparison. Intersex male bass were detected at three of the four RGB sites from which gonads were available for examination; none (of only three examined) were observed in fish from Station 514, and gonad samples were not obtained from Station 63 male bass. We did not detect ovotestis in male carp from any of the RGB sites investigated. Ovotestis was detected in male bass from Stations 16, 512, and 513, and exclusively in largemouth bass; however, only one male smallmouth bass (from Station 514) was examined. In the MRB, ovotestis was detected in male bass of both species, but a high percentage ( $>50 \%)$ of affected fish was only observed in smallmouth bass at one site and none were detected in carp (McDonald et al., 2002). Baldigo et al. (2000) detected similar percentages of both largemouth and smallmouth bass at some Hudson River (New York) sites, but no intersex males were reported among the largemouth bass exposed to paper mill effluents by Sepúlveda et al. (2001, 2003).

Differences between carp and bass were evident for GSI; gonads constituted a substantially greater proportion of the total body mass in carp. However, except for a few fish, among-station differences were not evident after accounting for stage and collection date differences. In contrast, Wainwright et al. (2001) noted a positive correlation between GSI carcass concentrations of $p, p^{\prime}$-DDE and plasma androgen concentrations in male carp from resacas and settling basins in the lower Rio Grande valley. Changes in GSI have been reported in fish exposed to a variety of endocrine-modulating substances (e.g., Sepúlveda et al., 2001; Orlando et al., 2004); however, such differences may only become apparent as the spawning season approaches.

Vitellogenin in female fish is important as a nutrient for developing embryos. Overall, plasma vtg concentrations in female carp and bass did not appear abnormally low at any station. In male fish, elevated vtg may indicate exposure to xenoestrogens. Concentrations in two male carp from Station 511 were within the range of early-to mid-vitellogenic females (Table 6), a condition that has been associated with the exposure of male fish to exogenous estrogens in both field and laboratory studies (e.g., Folmar et al., 1996, 2001; Gimeno et al., 1997; Jobling et al., 1998). In addition, and keeping with the findings of most field studies (e.g., Goodbred et al., 1997; Lee et al., 2000; McDonald et al., 2002; Solé et al., 2002, 2003), none of the vitellogenic male carp were intersex. These findings contrast with those of a study of reproductive biomarkers in another European cyprinid (roach, Rutilus rutilus) collected near sewage treatment plants in which the proportion of intersex fish and the severity of the intersex condition were greater than reference fish, and the mean vtg concentration of the intersex males was intermediate between that of males and female controls (Jobling et al., 1998). In RGB bass, relatively high vtg concentrations in stage1 females and an elevated concentration in one male 
were detected at Station 513. The vtg concentration in this latter fish as well as those in the two male carp from Station 511 were within the range of concentrations shown to induce pathological changes in the livers of male fish exposed to $17-\beta$ estradiol in laboratory studies (Folmar et al., 2001).

\section{Conclusions}

Overall, and as reported for the MRB (Schmitt, 2002a) we saw no evidence indicating that fish in the RGB had been exposed to extremely high concentrations of toxic chemicals. Rather, the biomarker data for the lower RGB stations are consistent with subtle responses of the fish to chronic contaminant exposure. Previous studies (Davis et al., 1995; TNRCC, 1997) indicated the potential for toxic chemical impacts on biota in the Arroyo Colorado at Harlingen and in the Rio Grande at Brownsville, and contaminants from agriculture and energy extraction have been recognized as threats to wildlife in the lower RGB (White et al., 1983; Gamble et al., 1988; Mora, 1997; Mora et al., 1997). Our findings continue to support these conclusions and concerns; although lower than in the past, concentrations of several organochlorine and elemental contaminants were great enough to represent a potential hazard to populations of the most sensitive fish-eating wildlife species.

The human population of the RGB is growing rapidly, which will expand both the magnitude and scope of chemicals released in the region and the demand for already limited amounts of water. Although our data and that of other programs and studies have documented declining concentrations of some persistent contaminants, the continuing growth of irrigated agriculture in the RGB may further exacerbate the leaching of toxic trace elements into ground and surface waters. Concentrations of $\mathrm{Hg}$ also remain at potentially hazardous concentrations in the mainstem impoundments, which are inhabited by susceptible wildlife species including the federally listed bald eagle (Haliaeetus leucocephalus) and interior least tern (Sterna antillarum athalassos). In addition, contemporary-use agricultural pesticides such as atrazine, chlorpyrifos, and diazinon have been detected at potentially problematic concentrations in the lower RGB by other programs. Concentrations of only a few of these latter compounds exceeded current standards and criteria (Schmitt et al., 2004); however, concentrations may rise in the future due to changing agricultural practices, urban growth, and declining water availability. Subtle responses to these and other chemicals may occur at concentrations lower than current standards, and their cumulative effects are largely unknown (e.g., Scholz et al., 2000; McDonald et al., 2000). Overall, results from the suite of biological endpoints we measured were consistent with chronic exposure to chemicals at the lower basin sites. Such responses would also be expected to increase in magnitude with increasing pesticide concentrations, which might ultimately threaten fish populations.

Mora and Wainwright (1998) urged further monitoring of the RGB due to limited historical data, increasing development, and a dearth of information on activities and contaminant releases in northern Mexico. Our findings also support this recommendation. Continued monitoring provides the basis for identifying consistently degraded sites as well as those with emerging problems, and for evaluating the success of remedial activities. The weight-of-evidence approach we employed is useful for detecting diffuse effects, especially those involving cumulative exposure to short-lived chemicals and those that do not accumulate in fish. Focused investigations are also necessary to document chemical sources and processes, cause-effect relationships, and possible roles of factors other than contaminants. The results of this study should therefore be combined with those of related investigations (e.g., Schmitt, 2002a) and with water and sediment data from other programs to create a data set spanning a wide range of exposure conditions to many contaminants. These data should be analyzed using more powerful statistical methods (e.g., Adams et al., 1994) to identify potential causal relationships between contaminant exposure and responses at multiple levels of biological organization that can be explored through controlled field and laboratory investigations.

\section{Acknowledgments}

This study was conducted jointly by the USGS, the U.S. Fish and Wildlife Service (USFWS), and cooper- 
ators at the University of Florida, Gainesville (UF). Many individuals representing USGS, USFWS, UF, and other organizations contributed substantially. C. Bunck (USGS) managed the BEST program during most of the study, and S. Finger (USGS) coordinated much of the work. M. Wilson and C. Lee (both USFWS) supervised field activities. Chemical analyses were conducted at laboratories operated by Mississippi State University and the Research Triangle Institute through contracts managed by the USFWS; J. Moore and P. McDonald (USFWS) facilitated this part of the study. Laboratory analyses for biomarkers were conducted by D. Nicks, S. Birke, D. Bowling, K. Spring, and E. Frankenberry (all USGS), and K. Kroll (UF). A. Donahue (USGS), J. Smith (USFWS), D. Buckmeier (Texas Parks and Wildlife, Ingram, TX) and E. Buckner (Missouri Department of Conservation, Columbia, MO) aged the fish. A. Donahue also managed the data and prepared report graphics. The study was partly funded through a Research Work Order with the USGS-Florida Cooperative Fish and Wildlife Research Unit at UF; F. Percival, Unit Leader, and T. Gross (USGS), facilitated this agreement. P. Anderson (USGS) prepared the map. B. Wright (Oregon Department of Fish and Game, Corvallis, OR) and M. Ellersieck (University of Missouri-Columbia) assisted with statistical analyses. D. Buckler, M. Mora, J. Moring, and C. Caldwell (all USGS); J. Whyte (AScI, c/o USGS); and J. Lusk (USFWS) reviewed all or parts of earlier versions of the RGB report and provided information. R. Lipkin (USGS) managed the bibliographic database. Comments of anonymous reviewers were very helpful.

\section{References}

Ackerman GE, Brombacher E, Fent K. Development of a fish reporter gene system for the assessment estrogenic compounds and sewage treatment plant effluents. Environ Toxicol Chem 2002;21:1864-75.

Adams SM, McLean RB. Estimation of largemouth bass, Micropterus salmoides Lacepede, growth using the liver somatic index and physiological variables. J Fish Biol 1985; 26:111-26.

Adams SM, Brown AM, Goede RW. A quantitative health assessment index for rapid evaluation of fish condition in the field. Trans Am Fish Soc 1993;122:63-73.

Adams SM, Ham KD, Beauchamp JJ. Application of canonical variate analysis in the evaluation and presentation of multi- variate biological response data. Environ Tox Chem 1994; 13:1673-83.

Aguillar A. Relationships of DDE/DDT in marine mammals to the chronology of DDT input into the ecosystem. Can J Fish Aquat Sci 1984;21:840-4.

Anderson DW, Jehl Jr JR, Risebrough RW, Woods Jr LA, DeWeese WG, Edgecomb WG. Brown pelicans: improved reproduction off the southern California coast. Science 1975;190:806-8.

Ankley GT, Tillitt DE, Giesy JP, Jones PD, Verbrugge DA. Bioassay-derived 2,3,7,8-tetrachlorodibenzo- $p$-dioxin equivalents in PCB-containing extracts from the flesh and eggs of Lake Michigan chinook salmon (Oncorhynchus tshawytscha) and possible implications for reproduction. Can J Fish Aquat Sci 1991;48:1685-90.

Au DWT, Chen P, Pollino CA. Cytological changes in association with ethoxyresorufin $O$-deethylase induction in fish upon dietary exposure to benzo[a]pyrene. Environ Toxicol Chem 2004;23:1043-50.

Baldigo B, Sloan RJ, Smith SB, Keane DP. Polychlorinated biphenyls and endocrine disruption in fish from the Hudson River, New York, USA. Society of Environmental Toxicology and Chemistry, 3rd World Congress, "Global Environmental Issues in the 21st Century", May 21-25, Brighton, UK, poser, viewable online at http://ny.water.usgs.gov/pubs/posters/ pcbendocrine.pdf.

Baumann PC, Mac MJ, Smith SB. Tumor frequencies in walleye and brown bullhead and sediment contaminants in tributes of the Laurentian Great Lakes. Can J Fish Aquat Sci 1991;48:1804-10.

Bidleman TF, Wall MD, Muir DCG, Stern GA. Selective accumulation of polychlorocamphenes in aquatic biota from the Canadian arctic. Environ Toxicol Chem 1993;12:701-10.

Blazer VS. Histopathological assessment of gonadal tissue in wild fishes. Fish Physiol Biochem 2002;26:85-101.

Blazer VS, Dethloff GM. Immune system indicators. In: Schmitt CJ, Dethloff GM, editors. Biomonitoring of Environmental Status and Trends (BEST) Program: Selected Methods for Monitoring Chemical Contaminants in Aquatic Ecosystems. U.S. Geological Survey Information and Technology Report USGS/BRD 2000-0005. p. 25-30. Columbia, MO.

Blazer VS, Wolke RE, Brown J, Powell CA. Piscine macrophage aggregate parameters as health monitors: effect of age, sex, relative weight, season and site quality in largemouth bass (Micropterus salmoides). Aquat Toxicol 1987;10:199-215.

Blazer VS, Dethloff GM, Wright B. Chapter 3, Fish health indicators. In: Schmitt CJ, editor. Biomonitoring of Environmental Status and Trends (BEST) Program: Environmental Contaminants and Their Effects on Gish in the Mississippi River Basin. U.S. Geological Survey Biological Science Report USGS/BRD/BSR 2002-0004, p. 89-134 Columbia, MO.

Bloom NS. On the chemical form of mercury in edible fish and marine invertebrate tissue. Can J Fish Aquat Sci 1992;49: $1010-7$.

Blus LJ. DDT, DDD, and DDE in birds. In: Beyer WN, Heinz GH, Redmon-Norwood AW, editors. Environmental Contaminants in Wildlife: Interpreting Tissue Concentrations. Boca Raton, FL: Lewis Publishers; 1996. p. 49-71. 
Brown SB, Adams BA, Cyr DG, Eales JG. Contaminant effects on the teleost fish thyroid. Environ Toxicol Chem 2004;23: $1680-701$.

Brumbaugh WG, Krabbenhoft DP, Helsel DR, Wiener JG, Echols KR. A national pilot study of mercury contamination of aquatic ecosystems along multiple gradients: bioaccumulation in fish. US Geological Survey Biological Science Report USGS/BRD/BSR-2001-0009, 2001. 25p. Columbia, MO.

Caldwell CA, Arnold MA, Gould WR. Mercury distribution in blood, tissues, and feathers of double-crested cormorant nestlings from arid-lands reservoirs in south central New Mexico. Arch Environ Contam Toxicol 1999;36:456-61.

Canavan CM, Caldwell CA, Bloom NS. Discharge of methylmercury-enriched hypolimnetic water from a stratified reservoir. Sci Total Environ 2000;260:159-70.

Carlander KD. Handbook of Freshwater Fishery Biology. Ames: Iowa State University Press; 1969. 752p.

Carlander KD. Life history data on centrarchid fishes of the United States and Canada. Ames: Iowa State University Press; 1977. $431 \mathrm{p}$.

Carter LF, Anderholm SK. Water-quality assessment of the Rio Grande Valley, Colorado, New Mexico, and Texas - organic compounds and trace elements in bed sediment and fish tissue, 1992-1993. US Geological Survey Water-Resources Investigations Report 97-4001, 1997. 23p.

Coughlan DJ, Baker BK, Cloutman DG, Rash WM. Application and modification of the fish health assessment index used for largemouth bass in the Catawba River, North Carolina-South Carolina. Am Fish Soc Symp 1996;16:73-84.

Coyle JJ, Buckler DR, Ingersoll CG, Fairchild JF, May TW. Effects of dietary selenium on the reproductive success of bluegills (Lepomis macrochirus). Environ Toxicol Chem 1993; 12:551-65.

Cross JN, Hose JE. Evidence for impaired reproduction in white croaker (Genyonemus lineatus) from contaminated areas off southern California. Mar Environ Res 1988;24:185-8.

Davis, JR, Kleinsasser, L, Cantu, R. Toxic contaminants survey of the Lower Rio Grande, lower Arroyo Colorado, and associated coastal waters, Texas Natural Resource Conservation Commission, Austin, 1995. AS-69, 127 p.

Denslow ND, Chow MC, Kroll KJ, Green L. Vitellogenin as a biomarker of exposure for estrogen or estrogen mimics. Ecotoxicology 1999;8:385-98.

Dethloff GM, Schmitt CJ. Condition factor and organo-somatic indices. In: Schmitt CJ, Dethloff GM, editors. Biomonitoring of Environmental Status and Trends (BEST) Program: Selected Methods for Monitoring Chemical Contaminants and their Effects in Aquatic Ecosystems US Geological Survey Information and Technology Report USGS/BRD 2000-0005, 2000. p. 13-8. Columbia, MO.

Drevnick PE, Sandheinrich MB. Effects of dietary methylmercury on reproductive endocrinology of fathead minnows. Environ Sci Technol 2003;37:4390-6.

Environmental Health Perspectives (EHP). Mexico moves to phase out DDT and chlordane. Environ Health Perspec 1997; 105:790-1.
Finney DJ. Statistics for biologists. New York: Chapman and Hall; 1980. 165p.

Fjeld E, Haugen TO, Vollestad LA. Permanent impairment in the feeding behavior of grayling (Thymallus thymallus) exposed to methylmercury during embryogenesis. Sci Total Environ 1998; 213:247-54.

Folmar LC, Denslow ND, Rao V, Chow M, Crain DA, Enblom J, et al. Vitellogenin induction and reduced serum testosterone concentrations in feral male carp (Cyprinus carpio) captured near a major metropolitan sewage treatment plant. Environ Health Perspec 1996;104:1096-101.

Folmar LC, Gardner GR, Schreibman MP, Magliulo-Cepriano L, Mills LJ, Zaroogian G, et al. Vitellogenin-induced pathology in male summer flounder (Paralichthys dentatus). Aquat Toxicol 2001;51:431-41.

Fournie JW, Summers JK, Weisberg SB. Prevalence of gross pathological abnormalities in estuarine fish. Trans Am Fish Soc 1996;125:581-90.

Fournie JW, Summers JK, Courtney LA, Engle VD, Blazer VS. Utility of splenic macrophage aggregates as an indicator of fish exposure to degraded environments. J Aquat Anim Health 2001;13:105-16.

Gamble LR, Jackson G, Maurer TC. Organochlorine, trace element, and petroleum hydrocarbon contaminants investigation of the lower Rio Grande Valley, Texas, 1985-86. US Fish and Wildlife Service, Ecological Services, Region 2. TX: Corpus Christi; 1988. 34pp.

Giesy JP, Bowerman WW, Mora MA, Verbrugge DA, Othoudt RA, Newsted JL, et al. Contaminants in fishes from Great Lakesinfluenced sections and above dams of three Michigan rivers: III Implications for health of bald eagles. Arch Environ Contam Toxicol 1995;29:309-21.

Gillespie RB, Baumann PC. Effects of high tissue concentrations of selenium on reproduction by bluegills. Trans Am Fish Soc 1986;115:208-13.

Gimeno S, Komen H, Venderbosch PWM, Bowmer T. Disruption of sexual differentiation in genetic male common carp (Cyprinus carpio) exposed to an alkylphenol during different life stages. Environ Sci Technol 1997;31:2884-90.

Gingerich WH. Hepatic toxicology of fishes. In: Weber LJ, editor. Aquatic toxicology. New York: Raven Press; 1982. p. $55-105$.

Goodbred SL, Gilliom RJ, Gross TS, Denslow ND, Bryant WL, Schoeb TR. Reconnaissance of 17ß-estradiol, 11-ketotestosterone, vitellogenin, and gonad histopathology in common carp of United States streams: potential for contaminant-induced endocrine disruption. US Geological Survey, Open-File Report 96627, 1997. 47p.

Guillette Jr LJ, Gross TS, Arnold SF, McLachlan JA. Ecoestrogens and embryos-is this a scientific basis for concern? Anim Reprod Sci 1996;42:13-24.

Hamilton SJ. Review of selenium toxicity in the aquatic food chain. Sci Total Environ 2004;326:1-31.

Hamilton SJ, Holley KM, Buhl KJ, Bullard FA, Weston LK, McDonald SF. Toxicity of selenium and other elements in food organisms to razorback sucker larvae. Aquat Toxicol 2002;59:253-81. 
Hammer C, Braum E. Quantification of age pigments (lipofuscin). Comp Biochem Physiol 1988;90B:7-17.

Hedrick RP, Groff JM, Okihiro MS, McDowell TS. Herpesviruses detected in papillomatous skin growths of koi carp (Cyprinus carpio). J Wildl Dis 1990;26:578-81.

Hermanutz RO, Allen KN, Roush TH, Hedtke S. Effects of elevated selenium on bluegills (Lepomis macrochirus) in outdoor experimental streams. Environ Toxicol Chem 1992; $11: 217-24$.

Hill KT, Womersley CZ. Interactive effects of some environmental and physiological variables on fluorescent age pigment accumulation in brain and heart tissues of an aquatic poikilotherm. Environ Biol Fish 1993;37:397-405.

Hunter RG, Carroll JH, Butler JS. The relationship of trophic level to arsenic burden in fish of a southern Great Plains lake. J Freshw Ecol 1981;1:121-7.

Jarvinen AW, Ankley GT, 1999. Linkage of effects to tissue residues: development of a comprehensive database for aquatic organisms exposed to inorganic and organic chemicals. Pensacola, FL: Society of Environmental Toxicology and Chemistry (SETAC) Press; 1999. 364p.

Jobling S, Nolan M, Tyler CR, Brighty G, Sumpter JP. Widespread sexual disruption in wild fish. Environ Sci Technol 1998; 32:2498-506.

June FC. Atresia and year-class abundance of northern pike, Esox lucius, in two Missouri River impoundments. J Fish Res Board Can 1970;27:587-91.

June FC. Reproductive patterns in seventeen species of warmwater fishes in a Missouri River reservoir. Environ Biol Fish 1977; 2:285-96.

Kania HJ, O'Hara J. Behavioral alterations in a simple predatorprey system due to sublethal exposure to mercury. Trans Am Fish Soc 1974;103:134-6.

Karr JR. Assessment of biotic integrity using fish communities. Fisheries 1981;6:21-7.

Kohler A. Identification of contaminant-induced cellular and subcellular lesions in the liver of flounder (Platichthys flesus) caught at different polluted estuaries. Aquat Toxicol 1990; 16:271-94.

Kortet R, Vainikka A, Taskinen J. Epizootic cutaneous papilomatosis in roach Rutilus rutilus: sex and size dependence, seasonal occurrence and between-population differences. Dis Aquat Org 2002;52:185-90.

Lange TR, Royals HE, Connor LL. Influence of water chemistry on mercury concentration in largemouth bass from Florida lakes. Trans Am Fish Soc 1993;122:74-84.

Law RJ. Metals in marine mammals. In: Beyer WN, Heinz GH, Redmon-Norwood AW, editors. Environmental Contaminants in Wildlife: Interpreting Tissue Concentrations. Boca Raton, FL: Lewis Publishers; 1996. p. 357-76.

Lee KE, Blazer VS, Denslow ND, Goldstein RM, Talmage PJ. Use of biological characteristics of common carp (Cyprinus carpio) to indicate exposure to hormonally active agents in selected Minnesota streams. US Geological Survey Water-Resources Investigations Report 00-4202, 2000. 47p.

Lemly AD. Assessing the toxic threat of selenium to fish and aquatic birds. Environ Monit Assess 1996;43:19-35.
Lemly AD. Selenium Assessment in Aquatic Ecosystems. New York: Springer-Verlag; 2002. 160pp.

Leonard PM, Orth DJ. Application and testing of an index of biotic integrity in small, coolwater streams. Trans Am Fish Soc 1986;115:401-14.

Levings GW, Healy DF, Richey SF, Carter LF. Water-quality of the Rio Grande Valley, Colorado, New Mexico, and Texas, 199295. US Geol Surv Circ 1998;1162:39.

Lorenzen A, Kennedy SW. A fluorescence-based protein assay for use with a microplate reader. Anal Biochem 1993;214:346-8.

Luna LG. Histopahological methods and color atlas of special stains and tissue artifacts. Gaithersburg, MD: American Histolabs, Inc.; 1992. 767pp.

Matsche MA, Grizzle JM. Early changes in pigmented macrophages in head kidney of channel catfish infected with Aeromonas hydrophila. J Aquat Anim Health 1999;3:253-61.

McDonald KK, Gross TS, Denslow ND, Blazer VS. Reproductive indicators. In: Schmitt CJ, Dethloff GM, editors. Biomonitoring of Environmental Status and Trends (BEST) Program: Selected Methods for Monitoring Chemical Contaminants and their Effects in aquatic ecosystems US Geological Survey Information and Technology Report USGS/BRD 2000-0005, 2000. p. 30-42 Columbia, MO.

McDonald KK, Gross TS, Denslow ND, Densmore C, Blazer VS. Chapter 4, Reproductive biomarkers. In: Schmitt CJ, editor. Biomonitoring of Environmental Status and Trends (BEST) Program: Environmental Contaminants and their Effects on Fish in the Mississippi River basin. US Geological Survey Biological Science Report USGS/BRD/BSR 2002-0004, p. 135-70 Columbia, MO.

Mora MA. Transboundary pollution: persistent organochlorine pesticides in migrant birds of the southwestern United States. Environ Toxicol Chem 1997;16:3-11.

Mora MA, Wainwright SE. DDE, mercury, and selenium in biota, sediments, and water of the Rio Grande-Rio Bravo Basin, 1965-1995. Rev Environ Contam Toxicol 1998;158:1-52.

Mora MA, Lee MC, Penny JP, Schultz TW, Sericano JL, Clum NJ. Potential effects of environmental contaminants on recovery of the aplomado falcon in South Texas. J Wildl Manage 1997;61:1288-96.

Mora MA, Papoulias D, Nava I, Buckler DR. A comparative assessment of contaminants in fish from four resacas of the Texas, USA-Tamaulipas, Mexico border region. Environ Internat 2001;27:15-20.

Mora MA, Skiles R, McKinney B, Paredes M, Buckler D, Papoulias $\mathrm{DM}$, et al. Environmental contaminants in prey and tissues of the peregrine falcon in the Big Bend Region, Texas, USA. Environ Pollut 2002;116:169-76.

Moring JB. Use of semipermeable membrane devices (spmd) to assess occurrence and estimate water concentrations of selected organic compounds in the Rio Grande from Presidio to Brownsville, Texas. US Geological Survey Fact Sheet FS-10099, 1999. 6pp. Austin, TX.

Muir D, Braune B, DeMarch D, Norstrom R, Wagemann R, Lockhart L, et al. Spatial and temporal trends and effects of contaminants in the Canadian Arctic marine ecosystem A review. Sci Total Environ 1999;230:83-144. 
Nagahama Y. The functional morphology of teleost gonads. In: Hoar WS, Randall DJ, Donaldson EM, editors. Fish Physiology, vol. IX, Part A. Orlando, FL: Academic Press; 1983. p. 223-64.

Newell AJ, Johnson DW, Allen LK. Niagara River biota contamination project: fish flesh criteria for piscivorous wildlife. New York Department of Environmental Conservation, Division of Fish and Wildlife, Bureau of Environmental Protection, Albany Technical Report 87-3, 1987. 182p.

Nowak BF, Kingsford MJ. Exposure to Thiodan ${ }^{\circledR}$ results in lipofuscin accumulation in hepatocytes of the freshwater catfish Tandanus tandanus. Dis Aquat Org 2003;56:135-43.

Ong K, O’Brien TF, Rucker MD. Reconnaissance investigation of water quality, bottom sediment, and biota associated with irrigation drainage in the middle Rio Grande valley and Bosque del Apache National Wildlfe Refuge, New Mexico, 1988-89. US Geological Survey Water-Resources Investigations Report 91-4036, 1991. 113p. Albuquerque, NM.

Orlando EF, Kolok AS, Binzcik GA, GAtes JL, Horton MK, Lambright CS, et al. Endocrine-disrupting effects of cattle feedlot effluent on an aquatic sentinel species, the fathead minnow. Environ Health Perspec 2004;112:353-8.

Patiño R, Wainscott MR, Cruz-Li EI, Balakrishnan S, McMurry B, Blazer VS, et al. Effects of ammonium perchlorate on the reproductive performance and thyroid follicle histology of zebrafish. Environ Toxicol Chem 2003;22:1115-21.

Rodriguez JN, Oteme ZJ, Hem S. Comparative study of vitellogenesis of two African catfish species Chrysichthys nigrodigitatus (Claroteidae) and Heterobranchus longifilis (Clariidae). Aquat Living Resour 1995;8:291-6.

Sanders RE, Miltner RJ, Yoder CO, Rankin ET. The use of external deformities, erosion, lesions, and tumors (DELT anomalies) in fish assemblages for characterizing aquatic resources: a case study of seven Ohio streams. In: Simon TP, editor. Assessing the Sustainability and Biological Integrity of Water Resources Using Fish Communities. Boca Raton, FL: CRC Press; 1999. p. 225-46.

Schmitt CJ. Environmental contaminants. In: Mac MJ, Opler PA, Puckett-Haecker CE, Doran PD, editors. Status and Trends of the Nation's Biological Resources, vol. 1. Reston, VA: U.S. Department of the Interior, U.S. Geological Survey; 1998. p. $131-65$.

Schmitt CJ, editor. Biomonitoring of Environmental Status and Trends (BEST) Program: Environmental Contaminants and their Effects on Fish in the Mississippi River Basin. US Geological Survey Biological Science Report USGS/BRD/BSR 2002-0004, 2002a. 217pp. Columbia, MO.

Schmitt CJ. Organochlorine chemical residues in fish from the Mississippi River basin, 1995. Arch Environ Contam Toxicol 2002b;43:81-97.

Schmitt CJ. Concentrations of arsenic, cadmium, copper, lead, selenium, and zinc in fish from the Mississippi River basin, 1995. Environ Monit Assess 2004;90:289-321.

Schmitt CJ, Dethloff GM, editors. Biomonitoring of Environment Status and Trends (BEST) Program: Selected Methods for Monitoring Chemical Contaminants in Aquatic Ecosystem. US Geological Survey Information and Technology Report USGS/ BRD/ITR-2000-0005, 2000. 81pp. Columbia, MO.
Schmitt C.J., Blazer V.S., Dethloff G.M., Tillitt D.E., Gross T.S., DeWeese L.R., Smith SB, Goede RW, Bartish TA, Kubiak TJ. Biomonitoring of Environmental Status and Trends (BEST) Program: field procedures for assessing the exposure of fish to environmental contaminants. U.S. Geological Survey Information and Technology Report USGS/BRD-1999-007, Columbia, MO, 1999a, iv+35 pp+ appendices.

Schmitt CJ, Zajicek JL, May TW, Cowman DF. National Contaminant Biomonitoring Program: concentrations of organochlorine chemical residues and elemental contaminants in US freshwater fish, 1976-1986. Rev Environ Contam Toxicol $1999 b ; 162: 43-104$.

Schmitt CJ, Tillitt DE, Whyte JJ. Chapter 2 Accumulative contaminants, H4IIE bioassay-derived dioxin equivalents, and ethoxyresorufin $O$-deethylase (EROD) activity. In: Schmitt CJ, editor. Biomonitoring of Environmental Status and Trends (BEST) Program: Environmental Contaminants and their Effects on Fish in the Mississippi River basin, US Geological Survey Biological Science Report USGS/BRD/BSR 2002-0004; 2002. p. 27-87. Columbia, MO.

Schmitt CJ, Dethloff GM, Hinck JE, Bartish TM, Blazer VS, Coyle JJ, et al. Biomonitoring of Environmental Status and Trends (BEST) Program: Environmental Contaminants and their Effects on Fish in the Rio Grande Basin. US Geological Survey, Scientific Investigations Report 2004-5108, 2004. 117 pp. Columbia, MO.

Scholz NL, Truelove NK, French BL, Berejikian BA, Quinn TP, Casillas E, et al. Diazinon disrupts antipredator and homing behaviors in Chinook salmon (Oncorhynchus tshawytscha). Can J Fish Aquat Sci 2000;57:1911-8.

Schultz R, Hermanutz R. Transfer of toxic concentrations of selenium from parent to progeny in the fathead minnow (Pimephales promelas). Bull Environ Contam Toxicol 1990;45:568-73.

Sepúlveda MS, Ruessler DS, Denslow ND, Holm SE, Schoeb TS, Gross TS. Assessment of reproduction in largemouth bass (Micropterus salmoides) exposed to bleached/unbleached kraft mill effluents. Arch Environ Contam Toxicol 2001;41: $475-82$.

Sepúlveda MS, Quinn BP, Denslow ND, Holm SE, Gross TS. Effect of pulp and paper mill effluents on reproductive success of largemouth bass. Environ Toxicol Chem 2003;22: $25-213$.

Sharpe RD. Development of the mercury mining industry: TransPecos Texas. Mining and Mineral Resources Research Institute Mineral Resource Circular, vol. 64. Austin: University of Texas; 1980. 32p.

Solé M, Barceló D, Prote C. Seasonal variation of plasmatic and hepatic vitellogenin and EROD activity in carp, Cyprinus carpio, in relation to sewage treatment plants. Aquat Toxicol 2002;60:233-48.

Solé M, Raldua D, Piferrer F, Barceló D, Porte C. Feminization of wild carp, Cyprinus carpio, in a polluted environment: plasma steroid hormones, gonadal morphology and xenobiotic metabolizing system. Comp Biochem Physiol Part C Toxicol Pharmacol 2003;136:145-56. 
Sorensen EMB. Selenium accumulation, reproductive status, and histopathological changes in environmentally exposed redear sunfish. Arch Toxicol 1988;61:324-9.

Southworth GR, Rurner RR, Peterson MJ, Bogle MA. Form of mercury in stream fish exposed to high concentrations of dissolved inorganic mercury. Chemosphere 1995;30:779-87.

Stansley W, Roscoe DE. Environmental toxicology - chlordane poisoning of birds in New Jersey, USA. Environ Toxicol Chem 1999;18:2095-9.

Statistical Analysis System (SAS) Institute. SAS/STAT User's Guide, Version 8. 1999. Carey, NC, 3848 pp.

Texas Natural Resource Conservation Commission (TNRCC). Regional Assessment of Water Quality in the Rio Grande Basin, including the Pecos River, the Devils River, the Arroyo Colorado, and the Lower Laguna Madre. AS-34 TNRCC, Austin, TX, 1994a, 375 pp+appendices.

Texas Natural Resource Conservation Commission (TNRCC). Binational Study Regarding the Presence of Toxic Substances in the Rio Grande/Rio Bravo and its Tributaries along the Boundary Portion between the United States and Mexico. Final Report, September 1994, Austin, TX, 1994b, 245 pp+appendices.

Texas Natural Resource Conservation Commission (TNRCC), 1997. Second phase of the binational study regarding the presence of toxic substances in the Rio Grande/Rio Bravo and its tributaries along the boundary portion between the United States and Mexico, Final Report, Austin, TX. (two volumes).

Tillitt DE, Giesy JP, Ankley GT. Characterization of the H4IIE rat hepatoma cell bioassay as a tool for assessing toxic potency of planar halogenated hydrocarbons in environmental samples. Environ Sci Technol 1991;25:87-92.

Toppari J, Larsen J, Christiansen P, Giwercman A, Grandjean P, Guillette Jr LJ, et al. Male reproductive health and environmental xenoestrogens. Environ Health Perspect 1996;104:741-803.

Treasurer JW, Holliday FGT. Some aspects of the reproductive biology of perch Perca fluviatilis L: a histological description of the reproductive cycle. J Fish Biol 1981;18:359-76.

Tyler CR, Jobling S, Sumpter JP. Endocrine disruption wildlife: a critical review of the evidence. Crit Rev Toxicol 1998; 28:319-61.

Tysklind M, Tillitt D, Eriksson L, Lundgren K, Rappe C. A toxic equivalency factor scale for polychlorinated dibenzofurans. Fundam Appl Toxicol 1994;22:277-85.

U.S. Environmental Protection Agency (USEPA). National Study of Chemical Residues in Fish. EPA, vol. 823-R-92-008. Washington, DC: Office of Science and Technology; 1992 (two volumes). van den Berg M, Birnbaum L, Bosveld BTC, Brunström B, Cook B, Feeley M, et al. Toxic equivalency factors (TEFs) for PCBs, PCDDs, PCDFs for humans and wildlife. Environ Health Perspect 1998;106:775-92.

van den Heuvel MR, Servos MR, Munkittrick KR, Bols NC, Dixon DG. Evidence for a reduction of 2,3,7,8-TCDD toxic equivalent concentrations in white sucker (Catostomus commersoni) exposed to bleached kraft pulp mill effluent, following process and treatment improvements. J Great Lakes Res 1996;22:264-79.

Van Metre PC, Mahler BJ, Callender E. Water-quality trends in the Rio Grande-Rio Bravo basin using sediment cores from reservoirs. US Geological Survey, Fact Sheet FS-221-96, 8p. Austin, TX.

Wainwright SE, Mora MA, Sericano JL, Thomas P. Chlorinated hydrocarbons and biomarkers of exposure in wading birds and fish of the lower Rio Grande valley, Texas. Arch Environ Contam Toxicol 2001;40:101-11.

White DH, Mitchell CA, Kennedy HD, Krynitsky AJ, Ribick MA. Elevated DDE and toxaphene residues in fishes and birds reflect local contamination in the Lower Rio Grande Valley, Texas. Southwest Nat 1983;28:325-33.

Whyte JJ, Jung RE, Schmitt CJ, Tillitt DE. Ethoxyresorufin- $O$ deethylase (EROD) activity in fish as a biomarker of chemical exposure. Crit Rev Toxicol 2000;30:347-570.

Whyte JJ, Schmitt CJ, Tillitt DE. The H4IIE cell bioassay as an indicator of dioxin-like chemicals in wildlife and the environment. Crit Rev Toxicol 2004;34:1-83.

Wiener JG, Spry DJ. Toxicological significance of mercury in freshwater fish. In: Beyer WN, Heinz GH, Redmon-Norwood AW, editors. Environmental Contaminants in Wildlife: Interpreting Tissue Concentrations. Boca Raton, FL: Lewis Publishers; 1996. p. 297-339.

Wiener JG, Krabbenhoft DP, Heinz GH, Scheuhammer AM. Ecotoxicology of mercury. In: Hoffman DJ, Rattner BA, Burton Jr GA, Cairns Jr J, editors. Handbook of ecotoxicology, 2nd ed. Boca Raton, FL: Lewis Publishers; 2002. p. $409-63$.

Wilcox R. Concentrations of selected trace elements and other constituents in the Rio Grande and in fish tissue in the vicinity of Albuquerque, New Mexico, 1994 to 1996. US Geological Survey Open-File Report 97-667, 1997. 173p.

Yeardley Jr RB, Lazorchak JM, Paulsen SG. Elemental fish tissue contamination in northeastern US lakes: evaluation of an approach to regional assessment. Environ Toxicol Chem 1998; 17:1875-84. 